\title{
Depolarization calibration and measurements using the CANDAC Rayleigh-Mie-Raman lidar at Eureka, Canada
}

\author{
Emily M. McCullough ${ }^{1,2}$, Robert J. Sica ${ }^{1}$, James R. Drummond ${ }^{2}$, Graeme Nott ${ }^{2, a}$, Christopher Perro ${ }^{2}$, \\ Colin P. Thackray ${ }^{2}$, Jason Hopper ${ }^{2}$, Jonathan Doyle ${ }^{2}$, Thomas J. Duck ${ }^{2}$, and Kaley A. Walker ${ }^{3}$ \\ ${ }^{1}$ Department of Physics and Astronomy, The University of Western Ontario, 1151 Richmond St., \\ London, ON, N6A 3K7, Canada \\ ${ }^{2}$ Department of Physics and Atmospheric Science, Dalhousie University, 6310 Coburg Rd., \\ P.O. Box 15000, Halifax, NS, B3H 4R2, Canada \\ ${ }^{3}$ Department of Physics, University of Toronto, 60 St. George St., Toronto, Ontario, M5S 1A7, Canada \\ apresent address: Facility for Airborne Atmospheric Measurements, Building 146, Cranfield University, \\ Cranfield, MK43 0AL, UK
}

Correspondence to: Emily M. McCullough (emccull2@uwo.ca)

Received: 18 March 2017 - Discussion started: 6 April 2017

Revised: 17 September 2017 - Accepted: 22 September 2017 - Published: 10 November 2017

\begin{abstract}
The Canadian Network for the Detection of Atmospheric Change (CANDAC) Rayleigh-Mie-Raman lidar (CRL) at Eureka, Nunavut, has measured tropospheric clouds, aerosols, and water vapour since 2007 . In remote and meteorologically significant locations, such as the Canadian High Arctic, the ability to add new measurement capability to an existing well-tested facility is extremely valuable. In 2010, linear depolarization $532 \mathrm{~nm}$ measurement hardware was installed in the lidar's receiver. To minimize disruption in the existing lidar channels and to preserve their existing characterization so far as is possible, the depolarization hardware was placed near the end of the receiver cascade. The upstream optics already in place were not optimized for preserving the polarization of received light. Calibrations and Mueller matrix calculations are used to determine and mitigate the contribution of these upstream optics on the depolarization measurements. The results show that with appropriate calibration, indications of cloud particle phase (ice vs. water) through the use of the depolarization parameter are now possible to a precision of \pm 0.05 absolute uncertainty ( $\leq 10 \%$ relative uncertainty) within clouds at time and altitude resolutions of $5 \mathrm{~min}$ and $37.5 \mathrm{~m}$ respectively, with higher precision and higher resolution possible in select cases. The uncertainty is somewhat larger outside of clouds at the same altitude, typically with absolute uncertainty $\leq 0.1$. Monitor-
\end{abstract}

ing changes in Arctic cloud composition, including particle phase, is essential for an improved understanding of the changing climate locally and globally.

\section{Introduction}

Clouds influence Earth's radiation budget and thus its weather and climate. Clouds reflect sunlight (cooling), and trap heat from the ground (warming). The combined effect of these competing influences is poorly understood, especially in the Arctic, because it depends significantly on the structure and microphysical properties of the clouds and the environment in which the clouds exist (Curry et al., 1996). Ice clouds radiate differently than water clouds (Sun and Shine, 1994). Tropospheric clouds occur frequently in the Arctic, with liquid content found at all times of year, often within mixed-phase clouds (Intieri et al., 2002; Shupe, 2011). In order to develop models with improved fidelity of the cloud phase, more observational measurement data sets are required (Shupe, 2011), with phase transitions being of particular interest (Kalesse et al., 2016). Measurements of cloud particle phase (ice vs. water) are therefore necessary in order to more fully understand the radiation balance of the Arctic atmosphere. Liquid droplets can exist well below 
$0^{\circ} \mathrm{C}$, so cloud temperature is not sufficient to determine the phase of cloud particles (e.g. Shupe et al., 2008; Boer et al., 2009; Curry et al., 1996; Sassen, 1991; Westbrook and Illingworth, 2013). Lidar depolarization measurements, which discern cloudy regions containing spherical particles (i.e. liquid droplets) from those containing non-spheres (i.e. ice particles), are one method by which cloud particle phase may be examined (Schotland et al., 1971; Sassen, 2005; Bourdages et al., 2009).

The Polar Environment Atmospheric Research Laboratory (PEARL) is located in Eureka, Nunavut $\left(80^{\circ} \mathrm{N}, 86^{\circ} \mathrm{W}\right)$, in Canada's High Arctic. PEARL has more than 25 instruments dedicated to the in situ and remote sensing study of atmospheric phenomena at a latitude where few measurements are typically available. With climate changes amplified at such latitudes (Serreze and Barry, 2011), PEARL's measurements are a valuable contribution to global atmospheric and environmental science.

The Canadian Network for the Detection of Atmospheric Change (CANDAC) Rayleigh-Mie-Raman lidar (CRL) was installed at PEARL in 2007. It has since made measurements of visible and UV particulate backscatter coefficient, aerosol extinction, water vapour mixing ratio, and other quantities using its 355 and $532 \mathrm{~nm}$ lasers and comprehensive detection package (Doyle et al., 2011; Nott et al., 2012). Adding $532 \mathrm{~nm}$ linear depolarization capabilities to this instrument is an economical way to add additional capacity to study Arctic clouds, in concert with other instruments at PEARL such as the Millimetre Cloud Radar (Moran et al., 1998), the E-AERI interferometer (Mariani et al., 2012), and the Starphotometer (Baibakov et al., 2015). To preserve continuity in the long-term data sets from other CRL channels, no existing optics were altered or removed during the installation of the depolarization channels. Figure 1 is a diagram of the CRL's receiver, showing the seven original measurement channels and indicating the locations of the new pellicle beam splitter, Polarotor rotating Glan-Thomson prism, interference filter, focusing lens, and photomultiplier tube (PMT) of the $532.1 \mathrm{~nm}$ depolarization channel. CRL uses a single PMT to measure light of two polarization planes on alternate laser shots, with a laser repetition rate of $10 \mathrm{~Hz}$. This is similar to Platt (1977), which likewise had a polarizer in front of its PMT that could be rotated between each laser shot, but which had laser repetition rates between 0.2 and $1 \mathrm{~Hz}$. CRL's higher repetition rate means that the assumption of simultaneous measurements in both polarization planes is reasonable. As the original lidar optics were not chosen for their polarization properties, the optical design of the CRL has made the calibration of the depolarization measurements challenging.

\subsection{Depolarization lidar theory}

With the new depolarization capabilities, we aim specifically to investigate "the atmospheric phenomena which change the polarization state of the light received by a lidar relative to the state of the transmitted light" (Gimmestad, 2008). Depending on the optical qualities of the particles, a population of randomly oriented identical particles in the atmosphere should either (a) not change the polarization state of the light (i.e. all light from that population will be returned polarized parallel with respect to the state of the transmitted light) or (b) cause the light to become completely unpolarized on its return. There may be more than one population of particles present in any given scattering volume. The calculation to determine the change in polarization requires a ratio of the intensity of light which is returned unpolarized to the total intensity of light which is returned in any and all polarization states (Flynn et al., 2008; Gimmestad, 2008). Expressed in this manner, the quantity of interest is $d$, the depolarization parameter: the portion of the total light intensity $I$ which has become depolarized through scattering. Similar descriptions, called depolarization factor, are given as early as van de Hulst (1957). The depolarization parameter is defined as

$d=\frac{I_{\text {unpol. }}}{\left(I_{\text {pol. }}+I_{\text {unpol. }}\right)}$.

In the event that the atmosphere does not depolarize the beam, there will be no intensity returned with polarization different than the transmitted light, and therefore $d=0$. In the case of complete depolarization, $d=1$.

Because lidars measure signals from PMTs and not the backscattered light intensity directly, the equation for $d$ and must be reformulated in terms of lidar observables. Gimmestad (2008) demonstrates this development using Mueller matrix algebra, with normalized matrices. Two quantities are measured. The first is the signal in a channel which uses a polarization analyzer to admit light polarized parallel to the polarization plane of the transmitted laser beam (the "parallel" channel) and a signal in a channel which uses a polarization analyzer to admit light polarized perpendicularly to this plane (the "perpendicular" channel). In this way, in the absence of any complicating factors, for linearly polarized transmitted light, the parallel channel will be sensitive to half of the backscattered light which has been unpolarized during scattering $\left(\frac{1}{2} I_{\text {unpol. }}\right)$ and all light which remains polarized during scattering $\left(I_{\text {pol. }}\right)$. The second signal is that in the perpendicular channel, which will be sensitive only to half of the unpolarized light $\left(\frac{1}{2} I_{\text {unpol. }}\right)$ and none of the light which remains polarized when backscattered. In Gimmestad's paper, the signals in the receivers, $S$, are individually "assumed to be calibrated", but no further details about these calibrations are provided. Presumably, this assumption considers the combined effects of all optics upstream of the PMT and the gain of the PMT, acting together as a constant attenuation factor for each individual channel. If the factors differ between channels, the overall effect in the system as a whole is that of partial polarizer. 


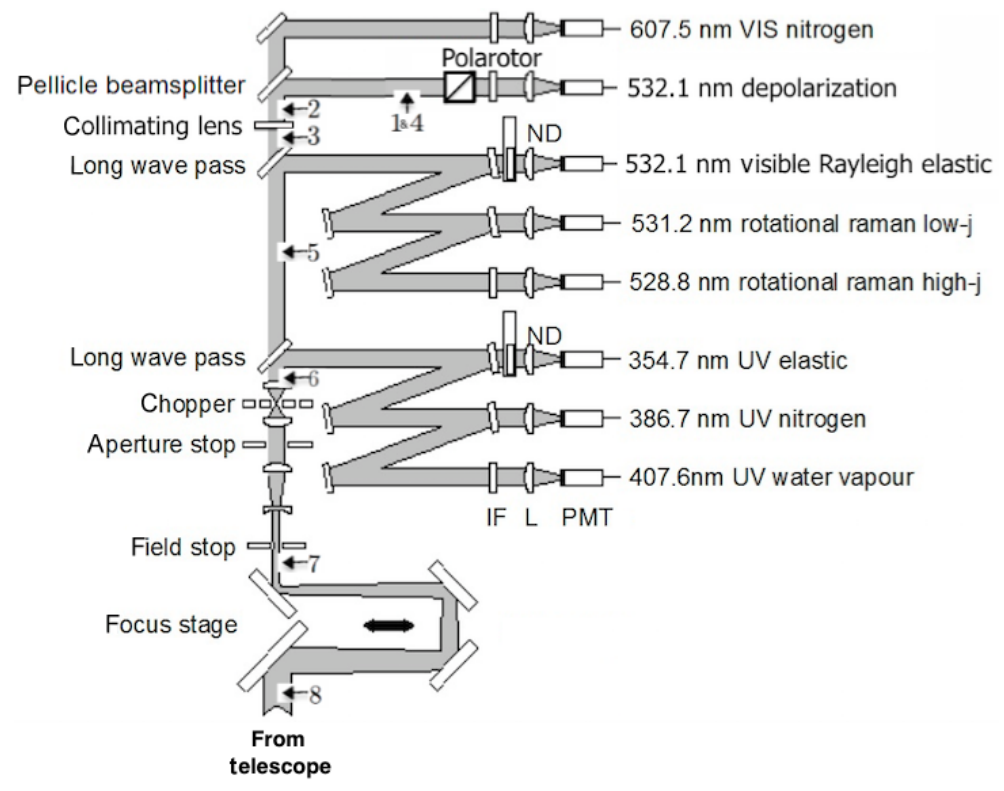

Figure 1. Diagram of CRL's receiver system, showing all seven existing measurement channels plus the newly installed depolarization hardware. The new hardware consists of the pellicle beam splitter, the Polarotor, and the interference filter, focusing lens, and PMT, to the right of the Polarotor. Numbers correspond to calibration test numbers from Table 1 and indicate the test locations of the depolarizing sheet used in those tests. The number "7" also marks the location of the calibration cube polarizer added to the system for the calibrations in Sect. 5.1, and " 8 " is also the location of the lamp and depolarization sheet during the polarized light tests in that section. During regular lidar sky measurements, neither the depolarizing sheet nor the calibration cube polarizer remain in the optical path. This figure is based on Fig. 2 of Nott et al. (2012).

Under these conditions, the equation for depolarization parameter is given as

$d=\frac{2 k S_{\perp}}{S_{\|}+k S_{\perp}}=\frac{2 k \frac{S_{\perp}}{S_{\|}}}{1+k \frac{S_{\perp}}{S_{\|}}}=\frac{2}{\frac{1}{k} \frac{S_{\|}}{S_{\perp}}+1}$,

in which $S_{\perp}$ is the signal measured by the perpendicular channel, $S_{\|}$is the signal measured by the parallel channel, and $k=\frac{G_{\|}}{G_{\perp}}$ is the depolarization calibration constant, where $G_{\|}$is the gain (or attenuation) of the parallel channel and $G_{\perp}$ is the gain (or attenuation) of the perpendicular channel. The third form for $d$ in Eq. (2) is easier to handle experimentally as each measurement appears only once and thus uncertainties may be considered uncorrelated.

Historically, "depolarization" has also referred to $\delta$, the depolarization ratio. This quantity is proportional to the ratio of the perpendicular signal $S_{\perp}$ to the parallel channel $S_{\|}$ (e.g. Hohn, 1969; Schotland et al., 1971; Liou and Schotland, 1971; Freudenthaler et al., 2009). The depolarization parameter $d$ is directly relatable to the expression for depolarization ratio, $\delta$, through the same signal measurements and the same calibration constant:

$\delta=k \frac{S_{\perp}}{S_{\|}}$.
The conversion between the quantities $d$ and $\delta$ is

$$
d=\frac{2 \delta}{(1+\delta)} \text {. }
$$

A variety of expressions for "depolarization" are described in Cairo et al. (1999). The $\delta$ described in the current paper is most closely related in meaning to the Pal and Carswell (1973) "volume linear depolarization ratio" cited therein, but it is not strictly equivalent because no claims are made here about the connection between $\delta$ and backscatter coefficient. Instead, $\delta$ is defined here only as a function of measured quantities. Gimmestad (2008) provides motivation for moving away from all $\delta$ descriptions, pointing out that $d$ is consistent with the rest of optical physics and scattering theory. Expressing depolarization as $d$ has since been adopted in such publications as Hayman and Thayer $(2009,2012)$ and Neely III et al. (2013). Results in the present paper will be provided in terms of both $d$ and $\delta$ so that readers working under either paradigm can readily make use of the figures and calculations.

The expressions for $d$ and $\delta$ of Eqs. (2) and (3) are all referred to in this paper as "traditional" in the sense that in each equation, a single $k$ value determines the calibration. 


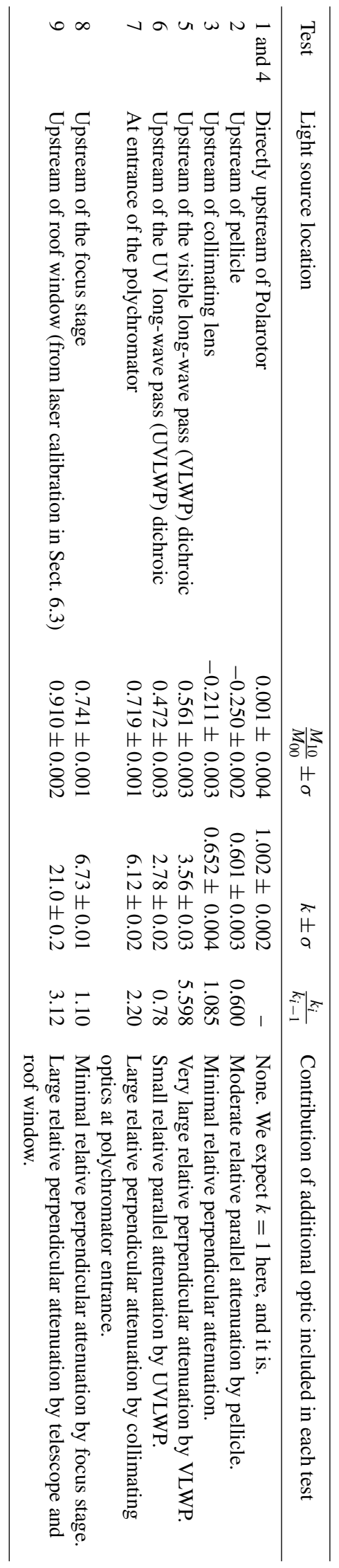

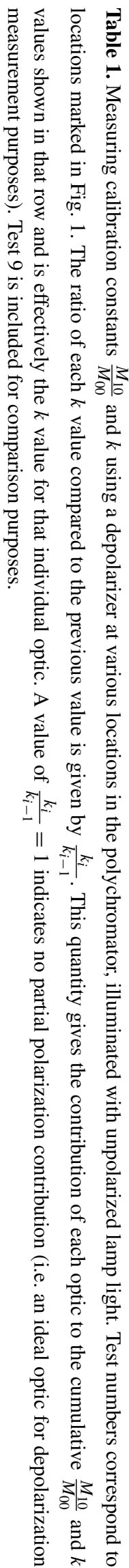

\subsection{Literature review of depolarization calibrations}

The calibration constant $k$ can be determined by introducing unpolarized light into the detector (i.e. setting $d=\delta=1$ ) or, equivalently, light polarized at $\pm 45^{\circ}$ with respect to each of the planes of polarization for the detectors (also sets $d=$ $\delta=1$ ) and measuring the signals in each channel. $k$ is then the ratio of the two signals. The location of calibration optics or lamps within the lidar determines how much of the system will be characterized through the calibration. The most strict meaning of $k$ is the ratio of gains of the detectors, if the polarization state of the calibration light is defined directly before the polarizing beam splitter. A wider interpretation for the meaning of $k$ can include relative gains resulting from other receiver optics if the polarization state is defined earlier in the receiver and can include relative gains of transmitter optics if the polarization state for the test is set within the transmitter. The laser is assumed to be completely linearly polarized. The orientation of the parallel and perpendicular polarization analyzers must be correctly set at $90^{\circ}$ to each other and oriented correctly with respect to the usual polarized returns from the transmitted laser beam before the calibrations for $k$ commence. Using $k$ as the only calibration factor ignores the possibility of any retarding and rotating effects which may exist in the transmitter and receiver, in all of the optical components. This is more likely to be appropriate for lidars which have few receiver optics before the polarization analyzer (e.g. Wang et al., 2009) and is less likely to be appropriate for lidars which have many receiver optics which are not optimized for polarization measurements, such as the CRL.

Some groups begin the calibration for $k$ with lidar returns from an atmospheric region which is assumed not to depolarize the light (or to depolarize only a known minimal amount as a result of molecular scattering) for the duration of the calibration. Then a half-waveplate is included in the receiver to control the orientation of the polarized backscattered light as it enters the detectors, aligning it at $\pm 45^{\circ}$ with respect to both polarization analyzers (Spinhirne et al., 1982; McGill et al., 2002; Alvarez et al., 2006; David et al., 2012; Wang et al., 2009; Freudenthaler et al., 2009; Neely III et al., 2013; Bravo-Aranda et al., 2013; Freudenthaler, 2016). This type of calibration is not sensitive to polarization effects in the transmitter optics or to any optics upstream of the halfwaveplate. This typically means omitting at least the telescope and sometimes more optics. A notable exception is Neely III et al. (2013), which has a waveplate optic in the roof window.

In an alternate version of the half-waveplate calibration, this waveplate may be placed in the transmitter to control the orientation of the plane of polarization of the laser light transmitted to the sky (Liu and Wang, 2013; Neely III et al., 2013; Freudenthaler, 2016; Bu et al., 2017). Eloranta and Piironen (1994) use a Pockels cell in their laser to the same effect. 
Locating the calibration optic in the transmitter is a method which includes as many optics as possible in the calibration.

All calibrations using polarized light must be concerned with obtaining the correct orientation of the waveplate (or other relevant optic). Spinhirne et al. (1982) and some lidar examples in Freudenthaler et al. (2009) arrange the waveplate as well as possible such that the output is at +45 and/or $-45^{\circ}$ and report the calibration measurements only from those specific angles. Other groups show improved results for $k$ by calculating its value at a variety of waveplate rotation angles, then using a fit to determine the optimal rotation angle from which to use the calibration values (Alvarez et al., 2006; Hayman and Thayer, 2009; Snels et al., 2009; Liu and Wang, 2013; Bu et al., 2017). Of those in the latter case, methods using both +45 and $-45^{\circ}$ measurements together (calibrations $90^{\circ}$ apart from one another) can have error terms which compensate well for one another in the event that the waveplate is misaligned by the same amount in each case (Freudenthaler et al., 2009, some systems in Freudenthaler, 2016).

Sassen and Benson (2001) use a different method for simulating a $d=\delta=1$ situation. They introduce unpolarized lamp light to their detector from the point of focus of the telescope. This calibration method is not sensitive to any polarization effects in the transmitter optics or the telescope, but the polarization state of the calibration light is well known, and there are no calibration optic rotation angles to introduce errors.

CRL calibrations for $k$ use a sheet of depolarizing material in the receiver. This can be placed at a variety of locations within the receiver. The results of these tests provide the motivation for this paper. When using a depolarizing sheet directly upstream of the Polarotor (see Fig. 1, locations 1 and 4; same location as half-waveplate used in e.g. Alvarez et al., 2006; Wang et al., 2009; Freudenthaler et al., 2009), we find that $k=1$. This is exactly as expected. Effectively, this is an estimate for the strict version of $k$ : the ratio of PMT gains and CRL uses the same PMT for each depolarization channel. Repeating the calibration measurements with a depolarizing sheet at the entrance to the receiver roof window suggest a value closer to $k=21.0 \pm 0.2$ for the whole CRL receiver (Sect. 6.3), indicating that optics upstream of the Polarotor are significantly polarizing. Clearly a more thorough instrument depolarization characterization is required for the CRL. If our optics are so highly polarizing, they may carry other optical consequences as well, which Eqs. (2) and (3) are insufficient to describe.

Various approaches are available in the literature to account for non-ideal depolarization lidar hardware, each with their own assumptions. Some calibrations are tests with a temporarily installed optic. These allow for calibration factors to be determined, which will then be applied to regular measurements made without the temporary calibration optic in place. Other calibrations consist of adjusting compensation optics (typically by adjusting their rotation angle) which will remain in the lidar during regular measurements. Some of these are the same optics used for the calculations of $k$. At present, the lidar of Neely III et al. (2013) seems most capable of a whole-system calibration. Their calibration optics exist in multiple places within the transmitter and multiple places within the receiver. The laser light can be rotated directly as it exits the laser, again as it exits the laboratory, again as it enters the telescope, and altered yet again as the light enters the PMTs. Further, the lidar was designed to make depolarization measurements, and optics could be selected and oriented with this in mind, as indicated in Hayman and Thayer (2012). Their liquid crystal variable retarder has some effects related to laboratory temperature which must be considered, but the authors have accounted for these. No other lidar of which we are aware has all of these capabilities. The more common calibrations each assess only some of the possible complications. Two are discussed briefly here.

First, Sassen and Benson (2001) allow calibration for the effect of angular misalignment between the transmitter and receiver planes of polarization in their measurements and calibrations. Other groups have introduced methods of optical compensation for such an angular misalignment: the half-waveplates used in the transmitters of Spinhirne et al. (1982), Liu and Wang (2013), and Bu et al. (2017), in the receivers of McGill et al. (2002), Wang et al. (2009), and Freudenthaler et al. (2009), and in both transmitter and receiver of Neely III et al. (2013) for the $k$ calibration remain in the lidars. During regular measurements, the optics are aligned such that a maximum of non-depolarized backscattered light is directed to the appropriate channel. To account for angular mismatch between transmitted and received planes of polarization, these calibrations generally depend on a "known" sky depolarization of aerosol-free molecular-only scattering (e.g. Platt, 1977) or liquid-dropletonly stratospheric clouds (e.g. Adachi et al., 2001, requiring additionally a total backscattering ratio measurement). Bravo-Aranda et al. (2016) and Freudenthaler (2016) analyze the effects of transmitter-receiver angular misalignment on uncertainties in the retrieved atmospheric depolarization values. The calculations therein are less relevant for CRL because both studies include the error that is induced by leaving an extra compensation optic in the lidar (the half-waveplate). The CRL (similarly to e.g. Alvarez et al., 2006) does not leave any calibration optics in the optical path during routine measurements. Thus, the uncertainties due to angular mismatch must be dealt with a different way. CRL carries out a Polarotor start delay test in clear sky (see Sect. 2.3) to ensure angular alignment between transmitted and received planes, and an assessment of uncertainty is carried out using a simple model.

Second, lidars using a polarizing beam splitter to separate received light to two separate PMTs have to account for different efficiencies for each plane of polarization in their parallel and perpendicular channels, one being reflected and the other transmitted through the beam splitter. These calcula- 
tions are integral to some recent works (Liu and Wang, 2013; Bravo-Aranda et al., 2016; Freudenthaler et al., 2009) but are not relevant for CRL, in which both parallel and perpendicular measurements are made using the transmitted beam of light through the Polarotor.

In contrast to the methods discussed to address these two complications, which tend to be dealt with individually, the CRL's approach in this paper is to determine the optical effects of the receiver as a whole. The recent papers on depolarization calibration are moving away from a scalar description, and are moving toward a vector description of light, with matrix algebra describing the optical effects of the sky (Kaul et al., 2004; Hayman and Thayer, 2009, using Mueller matrix algebra) and of the lidar itself (Biele et al., 2000; Hayman and Thayer, 2009, 2012; Neely III et al., 2013; Freudenthaler, 2016, using Mueller matrix algebra and $\mathrm{Bu}$ et al., 2017, using Jones matrices).

The Mueller matrix algebra upon which this technique relies was introduced as lectures and conference proceedings by Hans Mueller in the early 1940s (e.g. Mueller, 1946a, b, 1948). These and his previous works (Mueller, 1943a, b) remain difficult to obtain, and those available (e.g. in summary report Bush, 1946, which describes the design and use of the shutter described in Mueller, 1943a) do not explicitly demonstrate the matrix algebra. A better and more available source describing all of the Mueller matrix algebra in considerable detail is the thesis of Mueller's PhD student, Nathan Grier Parke III (Parke III, 1948).

In Hayman and Thayer (2012), there is a rigorous mathematical development of the Mueller matrices for lidar instrument optical contributions of various sorts. This is followed in a similar way by Freudenthaler (2016) and BravoAranda et al. (2016), which use Mueller matrix algebra to work out the expected signals for a sample of calibration and measurement situations, including errors. In those papers, and in Bu et al. (2017), many of the contributing matrices and vectors are considered to be standard forms for wellunderstood optics. In that sense, these works are a detailed forward-looking development intended to account for possible errors in known parameters (e.g. introducing a term for error in the rotation of transmitter with respect to receiver, and then determining it for their lidar). For CRL, we also take the Mueller matrix approach. We particularly follow the lead of Hayman and Thayer $(2009,2012)$ with regards to the mathematics, but with the opposite perspective: we initially presume to know nothing about the elements of the receiver optics Mueller matrix, and then we measure them.

\subsection{Mueller matrix calibration goals for CRL}

For the CRL, our approach is to use Mueller matrix mathematics to more fully diagnose the optical properties of CRL's receiver as a whole, similar to the approach taken by Di et al. (2016) and Liu and Wang (2013). We do not require the specific contributions of each receiver optic in order to un- derstand our measurements for $d$. We also do not need to split the matrices into equivalent standard optics (e.g. Hayman and Thayer, 2012, who describe optics as combinations of retarders, polarizers, etc.), nor do we need to do any of the specific examples given in Freudenthaler (2016), to adequately describe the CRL.

The first goal for this paper is to use Mueller matrix algebra to re-derive the equation for $d$, including calibration terms which describe the arbitrary optical effects of the upstream optics. These terms allow the collection of upstream optics to be represented using the most general single Mueller matrix possible (see Sect. 3.1, Eq. 12). We make no prior assumptions regarding rotation, retardation, or polarization properties of the optics. The beam splitter and laser polarizations are assumed to be ideal in our expressions.

The second goal is to use calibrations to measure the relevant matrix elements for the upstream optics which will indicate whether or not the overall impact of CRL's optics is that of a partial polarizer. If not, and it shows behaviour similar to that of a waveplate or a polarization rotator, then Eqs. (2) and (3) are insufficient to describe the depolarization parameter and depolarization ratio for CRL, and the full equations for $d$ from Sect. 3.1 will be required for routine measurements. The main tests introduce light of known polarization to the detector at a variety of rotation angles. This is generated by putting unpolarized light through a polarizing cube beam splitter. Some compromises must be made, as we must at times exclude the telescope and focus stage from our calibrations, similar to calibrations made by Platt (1977), Spinhirne et al. (1982), Sassen and Benson (2001), Wang et al. (2009), Bravo-Aranda et al. (2013), Bravo-Aranda et al. (2016), and others. With careful characterization of the remainder of the lidar receiver, we show that the overall contribution of the optics is indeed found to conform to that of a partial polarizer, rendering Eqs. (2) and (3) appropriate for CRL.

Third, we find the appropriate calibration constant $k$ for the whole receiver, including the telescope and focus stage, and using the laser as a light source, and using a sheet depolarizer to force all light entering the receiver to be unpolarized.

Fourth, we carry out additional unpolarized light tests to determine the contributions that individual optics make to the overall large $k$ value for CRL. The largest contributor to $k$ for CRL was found to be the visible long-wave pass (VLWP) filter (Sect. 7.2 and Table 1).

Finally, we demonstrate the use of the CRL's newly calibrated depolarization capability by showing some example measurements of ice clouds from 12 March 2013 in Sect. 8. The result for the CRL is a new depolarization data product tied into a scientifically significant long-term measurement record, all without compromise to the continued acquisition of the original types of data. To date, linear depolarization measurements have been made for four polar sunrise seasonal campaigns at Eureka: 2013, 2014, 2016, and 2017 (no measurements were obtained during 2015 because no operator was available). 


\section{Installation of depolarization hardware}

To make depolarization measurements, the lidar must be able to distinguish between backscattered light which is polarized parallel to the outgoing laser light, and that which is returned unpolarized. To this end, we make measurements in two orthogonal polarization planes: one parallel to the polarization plane of the outgoing laser light and the other perpendicular to this plane. While a polarizing beam splitter and two additional PMTs would accomplish this requirement, we opted to use a rotating polarizer which permits lidar returns in two orthogonal polarization planes to be measured by a single detector in an automated version of the measurement approach used in the very first depolarization lidars (Schotland et al., 1971). This design reduces the number of differences between the hardware of both depolarization channels because the backscattered light traverses identical optics and uses the same PMT. Given that the basic depolarization calculation is a ratio, having identical components means that many terms cancel out of the depolarization calculation.

The priority during installation of the polarization capability was not to impact any of the well-calibrated measurements in the other pre-existing lidar channels (Nott et al., 2012). No optics for the other channels were changed or removed during the installation of the depolarization channel, as these changes could have affected the other measurements.

\subsection{Polarotor}

The Licel Polarotor rotating polarizer (Licel GmbH, 2006) was designed specifically for multispectral detection systems such as that of CRL. The Polarotor acts as the master trigger for the lidar. Its $\alpha$-BBO Glan-Thompson prism is spun steadily at high speed, and a synchronization pulse from the built-in timing disk triggers the lidar system at $10 \mathrm{~Hz}$. This trigger signals the laser to fire and the detectors to record every time the prism rotates through $90^{\circ}$. The PMT is exposed to backscattered laser light which is polarized parallel to, and perpendicular to, the outgoing laser light, on alternate laser shots. Two recording buffers are used in the Licel transient recorder, one for parallel and one for perpendicular photocount profiles. The extinction ratio of the polarizer was characterized by the manufacturer to be $5 \times 10^{-5}$ or smaller (Licel $\mathrm{GmbH}, 2006$ ), leading to high-quality separation of the polarization states.

\subsection{Positioning of depolarization channel within CRL}

During manufacture, the CRL polychromator had two spare locations for potential expansion of the lidar. The depolarization channel was installed in the spare location between the $532 \mathrm{~nm}$ visible Rayleigh elastic channel and the $607 \mathrm{~nm}$ nitrogen channel (Fig. 1). This location is on the visible light side of the polychromator, but it suffers from being "downstream" of many optics. The original Chroma 580DCLP
VLWP filter was chosen in 2007 specifically to reflect as much $532 \mathrm{~nm}$ light into the original visible Rayleigh elastic channel as possible (approximately 97\%). Part of this reflected light is used in two subsequent visible channels at 531.2 and $528.7 \mathrm{~nm}$, and thus the requirement for maximum reflectivity is around $532 \mathrm{~nm}$ for the VLWP (Nott et al., 2012). The depolarization channel uses the small amount of residual $532 \mathrm{~nm}$ light which is transmitted through this VLWP filter on its way to the $607 \mathrm{~nm}$ channel, where it would normally be rejected by the $607 \mathrm{~nm}$ channel's interference filter.

During depolarization channel installation, a partially reflective optic was installed to redirect the residual $532 \mathrm{~nm}$ light into the depolarization channel, allowing the $607 \mathrm{~nm}$ light to continue on to the final PMT. The $607 \mathrm{~nm}$ channel optics were already well aligned and characterized at the time of depolarization installation (Doyle et al., 2011; Nott et al., 2012). Therefore, a regular plate beam splitter or dichroic mirror could not be used to pick off the light for the depolarization channel; this would have translated the transmitted $607 \mathrm{~nm}$ light too much, and the downstream channel would have had to be realigned. A $76 \mathrm{~mm}$ (3 in.) CVI-Melles

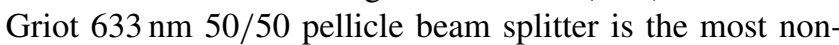
polarizing option available at $532 \mathrm{~nm}$ in reflectance, which still allows as much $607 \mathrm{~nm}$ light through as possible, with the smallest possible beam translation.

From the pellicle beam splitter, the $532 \mathrm{~nm}$ light travels into the Polarotor which is mounted on top of the polychromator. It sits on a $51 \mathrm{~mm}(2 \mathrm{in}$.) diameter beam tube which is $76 \mathrm{~mm}$ ( $3 \mathrm{in}$.) tall. The diameter of the light beam is slightly larger than $51 \mathrm{~mm}$ ( $2 \mathrm{in}$.) at the pellicle and, due to a collimating lens just upstream of the pellicle, is slightly converging. The Polarotor has an acceptance diameter of $20 \mathrm{~mm}$ and a full acceptance angle of $15^{\circ}$. By placing the Polarotor a sufficient distance from the pellicle, the entire beam is accepted by the Polarotor without the need for extra optics in between. It is also convenient to have the cables from the Polarotor to the electronics rack be accessible without the need to open the polychromator.

Above the Polarotor, there is a beam tube containing an interference filter, then a $25.5 \mathrm{~mm}$ ( 1 in.) diameter beam tube containing a $75 \mathrm{~mm}$ focusing plano-convex lens. Next, there is an adjustable focusing tube in which the Hamamatsu R7400-03 PMT is seated, which allows the active area of the PMT to be positioned at an appropriate distance from the focusing lens. The interference filter is Andover Corporation part number 532FS02-25, with a $25 \mathrm{~mm}$ diameter, BW $1 \pm 0.2 \mathrm{~nm}$ centred at $532.0 \pm 0.2 \mathrm{~nm}$, transmission greater than $45 \%$, and $1 \times 10^{-4}$ average blocking from X-ray to far infrared wavelengths (Andover Corporation, 2015). 


\subsection{Start delay calibration to define the "parallel" Polarotor rotation angle}

The Polarotor is set to spin at a $2.5 \mathrm{~Hz}(1.11 \mathrm{~ms}$ per degree of rotation). It can be set to trigger the laser from any polarizer angle. Timing in the Polarotor is controlled by a timing disk with four indicators $90^{\circ}$ apart, each of which can trigger the laser. Thus, once we set the rotation angle such that trigger 1 corresponds with "parallel", triggers 2,3 , and 4 correspond automatically to perpendicular, parallel, and perpendicular respectively, each $90^{\circ}$ of rotation after the other. One consequence of this arrangement is that although there may be error in the absolute angle during each measurement, the parallel and perpendicular channels are exactly $90^{\circ}$ apart.

In practice for the CRL, the reference rotation angle is controlled by the "start delay" time between the trigger pulse from the Polarotor and the time the laser fires. We perform a calibration to define the angle of rotation at which the Polarotor's prism needs to be in order for the parallel measurement channel to correspond to the polarized backscatter returns from the laser beam. In this manner we effectively align our polarizer with the plane of polarization of the laser as it is transmitted to the sky.

Start delay calibration measurements are made in a dark, clear sky, where the depolarization parameter should be approximately 0 . Measurements are taken in both depolarization channels for several minutes at each of many start delay settings. The optimal start delay setting occurs at the location of maximum contrast, where as much of the clear-sky signal as possible enters through the parallel channel and as little as possible enters via the perpendicular channel. In Eureka we work on a campaign basis, so it is not possible to wait months for the perfect clear day to do this measurement. We reduce the effects of any clouds, aerosols, or other depolarizing particle, by using only values from a certain altitude range of interest which does not include clouds and by using the polarization-independent Rayleigh elastic channel as a check to indicate times where our calibration may be invalid. It is helpful to divide the photon count rates of parallel and perpendicular by the count rates in the polarizationindependent channel to eliminate the effect of laser power variations. If the sky depolarization is not truly zero for this test, some systematic error will be induced in all subsequent calibrated measurements of sky depolarization.

From the test, we obtain two cosine curves of photocounts as a function of Polarotor start delay: one for parallel and the other for perpendicular, $180^{\circ}$ out of phase with one another. A fit to each curve allows us to find the start delay value.

Our start delay test is carried out in start delay steps of $2560 \mu$ s, with allowable start delay values between 20 and $419000 \mu$ s, as this has sufficient resolution to determine the correct start delay while requiring few enough settings that the calibration may be carried out during a single night. This test needs to be repeated any time the Polarotor is uninstalled or reinstalled into the polychromator.

\subsection{Effects of Polarotor rotation angle errors on depolarization measurements}

If we consider the angular start delay error to be equal to onehalf of our measurement step size of $2560 \mu$ s, corresponding to $1.408^{\circ}$ of error, we have the following errors in $d$ : the worst-case scenario is that for which $d=0$. There, a $1.408^{\circ}$ angle error corresponds to an error of \pm 0.0006 . For $d=1$, there is no uncertainty in $d$ from this source. For a typical value of $d=0.2$ the uncertainty is \pm 0.0005 .

Next, we investigated the effect of the rotation of the polarizer during each laser shot measurement and found that has negligible impact on our interpretation of $d$. Again considering the worst offending case, that in which $d=0$, a twoway maximum photon travel time for 16000 altitude bins is $0.0008 \mathrm{~s}$, during which the Polarotor rotates through $0.88^{\circ}$. The induced change in $d$ (in units of $d$ ) results in an uncertainty in $d$ such that $d=0.0000 \pm 0.0005$. The more diagnostic case $d=0.2$ results in $d=0.2 \pm 0.0004$.

When combined, the rotational errors of the Polarotor considered in this section contribute a maximum of total error of $2.288^{\circ}$, making the error from these sources approximately equal to \pm 0.003 for typical measured values of $d$. To put this in context, other errors (see Sect. 8) contribute \pm 0.1 to \pm 0.2 .

\section{Theory for depolarization calculations}

\subsection{Mueller matrix development}

A Mueller matrix approach was used to understand the signals being measured by the depolarization channel, with as few simplifications and assumptions as possible. In the case of atmospheric lidar operation, the original laser light is described as a Stokes vector which will be operated on by several optical elements, each of which can be described by a $4 \times 4$ matrix. We can thus determine the Stokes vector of the light which enters the PMTs and predict the signal which will be measured in each of our channels: $S_{\|}$(parallel channel measurement) and $S_{\perp}$ (perpendicular channel measurement).

In its most basic format, Mueller algebra functions as $\boldsymbol{L}^{\prime}=\mathbf{M} \boldsymbol{L}$, in which $\boldsymbol{L}$ and $\boldsymbol{L}^{\prime}$ are the Stokes vectors of incident and emerging radiation respectively, and $\mathbf{M}$ is the $4 \times 4$ real matrix effect of the instrument (Parke III, 1948). Stokes vectors add incoherently, and therefore the effect of any two components in series is equal to the product of their matrices. Thus, $\mathbf{M}$ can represent the product of two or more different optical matrices. Stated in terms of a matrix equation, the effects of the atmosphere $\mathbf{M}_{\mathrm{atm}}$ and of the lidar's receiver optics $\mathbf{M}_{\text {receiver }}$ on the emitted laser light are

$\boldsymbol{I}_{\text {measured }}=\mathbf{M}_{\text {receiver }} \mathbf{M}_{\text {atm }} \mathbf{M}_{\text {transmitter }} \boldsymbol{I}_{\text {laser }}$.

The CRL laser emits horizontally linearly polarized light, nominally $\boldsymbol{I}_{\text {laser }}=I_{\text {laser }}\left[\begin{array}{llll}1 & 1 & 0 & 0\end{array}\right]^{\prime}$. The laser intensity $I_{\text {laser }}$ varies shot to shot with such quantities as laser voltage and 
flashlamp age. Using alternate laser shots to build up 300 shot sums in each parallel and perpendicular in each one minute lidar scan allows these channels to measure simultaneously. Taking many shots into each sum allows the short timescale $(<1 \mathrm{~s})$ laser voltage variations to average out in each measurement. Therefore, $I_{\text {laser }}$ can be considered the same for both channels for each measurement pair. The polarization of the laser was measured with a cubic polarizing beam splitter on a kinematic mount placed in front of a power meter which is permanently mounted on the optical table. A mirror wheel redirects the beam into the power meter from a location partway through the transmitter. The precise location can be found in Nott et al. (2012). Thus, the polarization tests take into account all but four transmission optics on the optical table, as well as the one extra optic of the redirection mirror. This location is not ideal but is accessible in this installation. The first three elements of the resulting normalized Stokes vector $[I, Q, U, V]^{\prime}$ were calculated at two laser powers. At lower power, $[I, Q, U, V]^{\prime}=$ $[1 \pm 0,1.00 \pm 0.02,0.02 \pm 0.01, V \pm \sigma V]^{\prime}$. At higher power, $[I, Q, U, V]^{\prime}=[1 \pm 0,0.97 \pm 0.01,0.00 \pm 0.01, V \pm \sigma V]^{\prime}$. Our test was not directly sensitive to the value of $V$. Since $Q^{2}+$ $U^{2}+V^{2} \leq I^{2}$, we can surmise that $|V| \leq 0.243$. We consider $\boldsymbol{I}_{\text {laser }}=I_{\text {laser }}\left[\begin{array}{llll}1 & 1 & 0 & 0\end{array}\right]^{\prime}$ to be a reasonable laser Stokes vector for the calculations in this paper.

The optical backscattering effects of the atmosphere can be described as $\mathbf{M}_{\mathrm{atm}}$. Some authors assume ensembles of spheres and others the full normalized backscattering matrix for any shape of particle (Mishchenko and Hovenier, 1995). The latter is similar to the description in van de Hulst (1957), who describes a matrix specifically for backscattering of a cloud of asymmetrical particles with rotational symmetry, for which each particle has a mirror particle, and where there is no preferred orientation. Such is the case for the first matrix in Eq. (5), which can be fully described by two scalar values $a_{1}$ and $a_{2}$. Particularly interesting are changes in polarization during the scattering events. Gimmestad (2008) introduces variable $d$ such that the scattering matrix is normalized to have an intensity of 1 . For generality, we include here a gain factor, $b$. The gain factor is not stable long term, but for any given minute of data it will be constant for both channels. Under these assumptions $b$ cancels from the equations, demonstrating that the absolute scattering efficiency of the atmosphere has no effect on the measurements of depolarization.

$$
\begin{aligned}
\mathbf{M}_{\mathrm{atm}} & =\left(\begin{array}{cccc}
a_{1} & 0 & 0 & 0 \\
0 & a_{2} & 0 & 0 \\
0 & 0 & -a_{2} & 0 \\
0 & 0 & 0 & a_{1}-a_{2}
\end{array}\right) \\
& =b\left(\begin{array}{cccc}
1 & 0 & 0 & 0 \\
0 & 1-d & 0 & 0 \\
0 & 0 & d-1 & 0 \\
0 & 0 & 0 & 2 d-1
\end{array}\right)
\end{aligned}
$$

In Eq. (5) the quantity $d$ is the depolarization parameter for the atmosphere above the lidar, as described in Eq. (2), and describes the extent to which the transmitted light has been depolarized by the atmosphere.

The backscattered light already acted upon by $\mathbf{M}_{\text {atm }}$ passes through a first set of receiver optics, which are shared by both the parallel and perpendicular beam paths: telescope, focus stage, beam splitters, long-wave pass filters, etc. We account for these upstream optics together as a $4 \times 4$ matrix, $\mathbf{M}_{\text {upstream, but we make no assumptions as to the values of }}$ the matrix elements:

$\mathbf{M}_{\text {upstream }}=\left(\begin{array}{llll}M_{00} & M_{01} & M_{02} & M_{03} \\ M_{10} & M_{11} & M_{12} & M_{13} \\ M_{20} & M_{21} & M_{22} & M_{23} \\ M_{30} & M_{31} & M_{32} & M_{33}\end{array}\right)$.

There is also the possibility of an instrumental influence which varies with altitude $z$ : geometric overlap is such a term and is the largest influence in CRL's height-dependent calibration vector. Hence, all height-dependent variations will be attributed to geometric overlap, and we will subsequently call this the "overlap function", $\boldsymbol{O}(z)$. The overlap varies with changes to the lidar's alignment, so calibration values must be applied to measurements made within an appropriate time window (as alignment can change slowly with laboratory temperature) and must be redetermined after every routine alignment adjustment procedure.

Next, the light passes through the Glan-Thompson prism of the Licel Polarotor. This prism acts as either a horizontal or a vertical analyzing polarizer (for the parallel $\left(\mathbf{M}_{\|}\right)$ and perpendicular $\left(\mathbf{M}_{\perp}\right)$ measurement channels respectively) depending on its orientation at a particular time. The laser beam has linear polarization in the horizontal direction. During setup, the "parallel" analyzer position was also oriented such that it can be represented as a horizontal polarizer (by aligning the parallel direction with the direction of maximum signal in a low depolarization sky).

$$
\begin{aligned}
\mathbf{M}_{\|} & =\frac{1}{2}\left(\begin{array}{cccc}
1 & 1 & 0 & 0 \\
1 & 1 & 0 & 0 \\
0 & 0 & 0 & 0 \\
0 & 0 & 0 & 0
\end{array}\right) \text { and } \\
\mathbf{M}_{\perp} & =\frac{1}{2}\left(\begin{array}{cccc}
1 & -1 & 0 & 0 \\
-1 & 1 & 0 & 0 \\
0 & 0 & 0 & 0 \\
0 & 0 & 0 & 0
\end{array}\right)
\end{aligned}
$$

Finally, the light passes through the focusing lens and neutral density filter of the PMT and onto the PMT itself. The lens is axially symmetric, and the neutral density filter is used at normal incidence. Therefore, the effect of these optics on any incident light will be identical regardless of incoming polarization orientation. These optics are downstream of the analyzing polarizer, so any rotation of the plane of polarization by these optics will have no impact on the signal registered by the PMT and is therefore unimportant. The only 
effect of these optics is to reduce the amplitude of the signal by a constant factor regardless of incoming polarization. They are well described as a constant scalar factor. For lidars which have physically separate detectors or optical paths for the parallel and perpendicular channels, one such scalar factor will be required for each: $G_{\mathrm{PMT}_{\|}}$and $G_{\mathrm{PMT}_{\perp}}$. These terms are identical and cancel out of the equations below for CRL but will be included explicitly in the equations below as long as possible for generality.

These Mueller matrices combine to make an overall equation for each channel which describes the action of all optical components on the light and results in Stokes vectors $\boldsymbol{I}_{\|}$ (shown in full in Eq. 8) and $\boldsymbol{I}_{\perp}$ (which differs from $\boldsymbol{I}_{\|}$only by two minus signs in the polarizer matrix):

$$
\begin{aligned}
& \boldsymbol{I}_{\|}=\frac{G_{\mathrm{PMT}_{\|}}}{2}\left(\begin{array}{llll}
1 & 1 & 0 & 0 \\
1 & 1 & 0 & 0 \\
0 & 0 & 0 & 0 \\
0 & 0 & 0 & 0
\end{array}\right)\left(\begin{array}{llll}
M_{00} & M_{01} & M_{02} & M_{03} \\
M_{10} & M_{11} & M_{12} & M_{13} \\
M_{20} & M_{21} & M_{22} & M_{23} \\
M_{30} & M_{31} & M_{32} & M_{33}
\end{array}\right) \\
& b O_{\| \perp}(z)\left(\begin{array}{cccc}
1 & 0 & 0 & 0 \\
0 & 1-d & 0 & 0 \\
0 & 0 & d-1 & 0 \\
0 & 0 & 0 & 2 d-1
\end{array}\right) I_{\text {laser }}\left(\begin{array}{l}
1 \\
1 \\
0 \\
0
\end{array}\right) \text {. }
\end{aligned}
$$

The signal $S_{||}$measured by the lidar is the intensity element of the Stokes vector $\boldsymbol{I}_{\|}$:

$$
\begin{aligned}
S_{\|} & =\frac{G_{\mathrm{PMT}_{\|}} b O_{\| \perp}(z) I_{\text {laser }}}{2} \\
& \left(M_{00}+M_{10}+\left(M_{01}+M_{11}\right)(1-d)\right) .
\end{aligned}
$$

Similarly, $S_{\perp}$ is the intensity element of the Stokes vector $I_{\perp}$ :

$$
\begin{aligned}
& S_{\perp}=\frac{G_{\mathrm{PMT}_{\perp}} b O_{\| \perp}(z) I_{\text {laser }}}{2} \\
& \quad\left(M_{00}-M_{10}+\left(M_{01}-M_{11}\right)(1-d)\right) .
\end{aligned}
$$

Using the signals $S_{\|}$and $S_{\perp}$ from above for the complete matrix description of the lidar, we solve for the depolarization parameter $d$. The simplest method for combining lidar signals $S_{\|}$and $S_{\perp}$ into an equation for depolarization parameter comes from creating the quantity

$$
\frac{S_{\|}-S_{\perp}}{S_{\|}+S_{\perp}}=\frac{M_{10}+M_{11}(1-d)}{M_{00}+M_{01}(1-d)}
$$

realizing that $G_{\mathrm{PMT}_{\perp}}=G_{\mathrm{PMT}_{\|}}=G_{\mathrm{PMT}_{\| \perp}}$ for CRL because both channels are physically the same PMTs. Solving for $d$ yields Eq. (12):

$d=1-\frac{\frac{M_{10}}{M_{00}}\left(1+\frac{S_{\perp}}{S_{\|}}\right)-\left(1-\frac{S_{\perp}}{S_{\|}}\right)}{\frac{M_{01}}{M_{00}}\left(1-\frac{S_{\perp}}{S_{\|}}\right)-\frac{M_{11}}{M_{00}}\left(1+\frac{S_{\perp}}{S_{\|}}\right)}$.

For calibration, we must determine the three instrument constants $\frac{M_{01}}{M_{00}}, \frac{M_{10}}{M_{00}}$, and $\frac{M_{11}}{M_{00}}$. Note that we do not require the $M_{x x}$ values individually nor do we need to know the laser intensity.

\subsection{Conditions under which the traditional equations are appropriate}

Under the mathematical conditions $M_{01}=M_{10}$ and $M_{11}=$ $M_{00}$, the traditional expression for $d$ (Eq. 2) is equivalent to the more complete expression for $d$ (Eq. 12). In the case that CRL meets these conditions, it will be acceptable to use Eq. (2) in further calculations for this lidar. The matrix form of $\mathbf{M}_{\text {upstream }}$ that is required for this condition has the effect of a partial polarizer on measurements of $d$ : it acts with gain $G_{\mathrm{up}_{\|}}$for light polarized in the parallel direction and $G_{\mathrm{up}_{\perp}}$ for that polarized perpendicularly. Under these simplified conditions, the relation between $k$ and $M_{x x}$ is

$\frac{G_{\mathrm{up}_{\|}}}{G_{\mathrm{up}_{\perp}}}=\frac{M_{00}+M_{01}}{M_{00}-M_{01}}=\frac{1+\frac{M_{01}}{M_{00}}}{1-\frac{M_{01}}{M_{00}}}=k$.

Calibrations described in Sect. 5 demonstrate that the $M_{01}=M_{10}$ and $M_{11}=M_{00}$ conditions are met for CRL, allowing Eq. (3) to be used for the calculation of depolarization ratio and Eq. (2) for depolarization parameter.

\section{Optics for generating polarized and depolarized light during calibrations}

Instrument calibration tests to determine $k$ and the $M_{x x}$ and $G_{x x}$ values for CRL are described in the following sections. To carry out these tests, some additional calibration optics must be temporarily added to the lidar.

1. Calibration cube polarizer: the generating polarizer is a Newport 10BC16PC.3 Pol cube beamsplitter, $532 \mathrm{~nm}$, $25.4 \mathrm{~mm}, \mathrm{Tp} / \mathrm{Ts}>1000: 1$, a linearly polarizing cubic prism. It is placed immediately downstream of the focus stage (location label 7 in Fig. 1) and is rotated by hand. The rotating mount has markings in $2^{\circ}$ steps, and there is about half a step of uncertainty in either direction, hence $\pm 1^{\circ}$ uncertainty in the rotation angles $\theta$.

The matrix describing the cube polarizer, allowing for an attenuation factor $G_{\text {cube }}$, is

$$
\mathbf{M}_{\text {cube }}=\frac{G_{\text {cube }}}{2}\left(\begin{array}{cccc}
1 & \cos 2 \theta & \sin 2 \theta & 0 \\
\cos 2 \theta & \cos ^{2} 2 \theta & \frac{1}{2} \sin 4 \theta & 0 \\
\sin 2 \theta & \frac{1}{2} \sin 4 \theta & \sin ^{2} 2 \theta & 0 \\
0 & 0 & 0 & 0
\end{array}\right) \text {. }
$$

2. Glassine waxed paper depolarizer: typically used to protect works of art, the depolarizing properties of Lineco Glassine (Lineco glassine acid-free tissue 16 in. $\times 20$ in. $(41 \mathrm{~cm} \times 51 \mathrm{~cm}), 12$ pack, product number $448-1626)$ were found to be highly satisfactory. As tested by our group, after one sheet of glassine the residual polarization is less than $1 \%$ (polarization $=0.009 \pm 0.006$ ). Full details of this characterization are available in McCullough (2015), chap. 4.6.2.1 "Calibration tests of the 
optical qualities of glassine waxed paper". The depolarizing properties were not affected by the product's exposure to damp or to wetting and subsequent drying out. This product was mounted in such a way as to be held relatively taut in a frame or held in place by other mechanical means, depending on the specific calibration test. See McCullough (2015) for photographs of the arrangements used for the calibrations in this paper. Glassine is highly scattering material, so when the entire roof hatch window is covered with it the received lidar signal is greatly reduced.

The matrix for a perfect depolarizer with an attenuation parameter $G_{\mathrm{gl}}$, which is applicable to a real depolarizing optic (in this case, glassine waxed paper), is

$$
\mathbf{M}_{\text {glassine }}=\frac{G_{\mathrm{gl}}}{2}\left(\begin{array}{cccc}
1 & 0 & 0 & 0 \\
0 & 0 & 0 & 0 \\
0 & 0 & 0 & 0 \\
0 & 0 & 0 & 0
\end{array}\right) \text {. }
$$

3. Industrial kitchen grade waxed paper: the depolarizing properties of this product were used for CRL calibrations before we were aware of glassine, which turned out to be the superior material. The depolarizing properties of various brands of waxed paper, and from batch to batch within a particular brand, vary widely; verification for each application is advisable. As for glassine, waxed paper is a highly scattering material which greatly reduces received lidar signals when they are measured with the waxed paper in place.

\section{Calibrations to determine whether traditional equations are acceptable}

Introducing polarized lamp or laser light to the detector provides the calibration values which indicate whether the simple equations are satisfactory for CRL, by testing whether the conditions $M_{01}=M_{10}$ and $M_{11}=M_{00}$ are satisfied.

\subsection{Physical setup of the rotating polarizer test used at CRL}

A light source (lamp or backscattered laser) is directed through two layers of glassine depolarizer sheet into a polarization-generating optic and through to the lidar's receiver system. The polarization generator is rotated through various angles $\theta$ with respect to the plane of polarization of the parallel channel, and signals are measured in the parallel and perpendicular channels as a function of this angle (Fig. 2). The glassine ensures that unpolarized light enters the polarizer, and thus we begin with equal numbers of photons exiting the polarizer regardless of its orientation.

Ideally, as many optics as possible are included after the polarization generating calibration optic, so that the contributions of as many "upstream optics" as possible are in- cluded in the Mueller matrix $\mathbf{M}_{\text {upstream during calibration. }}$ In practice, this is difficult to accomplish for practical reasons at CRL. A light source which illuminates the entire $1 \mathrm{~m}$ CRL telescope is available if we employ backscattered laser light. A $1 \mathrm{~m}$ depolarizing optic to initially depolarize all the backscattered light received at the roof hatch level is also available (glassine waxed paper sheets). The problem is that no feasible polarizing optic has the required properties. We require a $1 \mathrm{~m}$ diameter optic which can be held completely flat and which can survive the harsh outdoor conditions of Arctic winter. The polarizing optic must be able to be easily and repeatedly rotated to the appropriate orientation, and should have sufficient optical polarization quality. A variety of setups using sheet polarizers were attempted to overcome these problems, and none produced satisfactory results. The use of a smaller $25 \mathrm{~cm}$ diameter sheet polarizer installed in a smaller aperture above the lidar's telescope, with the rest of the entrance to the primary mirror masked also did not. Repeating the test with the sheet polarizer held between the telescope's tertiary mirror and the focus stage worked better (location label 8 in Fig. 1), but it still relied on a suboptimal optical quality sheet polarizer.

By removing the roof window, the lidar's telescope, and the focus stage from consideration in the calibration, the rotating polarizer test becomes possible at CRL. By the time the light reaches the entrance to the polychromator, the received light beam, originally $1 \mathrm{~m}$ diameter, is focused small enough to allow the use of the $25 \mathrm{~mm}$ polarizing cube beam splitter of high optical quality described in Sect. 4 . The cube beam splitter can be rotated precisely and is stably mounted on a kinematic rotation mount on a $51 \mathrm{~mm}$ ( 2 in.) beam tube which leads into the polychromator from the focus stage (location label 7 in Fig. 1).

The telescope and focus stage are being omitted in the test. If the lidar return were used as the light source, we could still take into account the height dependency of the optics. Light collected from different heights has different light paths and different incidence angles in the receiving modules (Freudenthaler, 2016), and this may have effects on the calibration. While there are in principle advantages to including any height-dependent effects, in practice the glassine waxed paper depolarizer attenuates the backscattered laser signal too much to be of use in the practical case for a laser of CRL's power in clear skies. Additionally, the atmospheric conditions would need to remain stable throughout the test. Thus, there is little advantage to using lidar returns as the light source; a current-stabilized constant lamp source provides more signal with better control of the experimental setup. It also does not rely on specific atmospheric conditions. The lamp is installed on the telescope frame such that it shines through a glassine depolarizing sheet held taut in a frame of foamcore between the tertiary telescope mirror and the focus stage (location label 8 in Fig. 1). The resulting unpolarized light is sent through the focus stage, then through the cube polarizer, which produces the linearly po- 
(a) Polarized light calibration: parallel channel

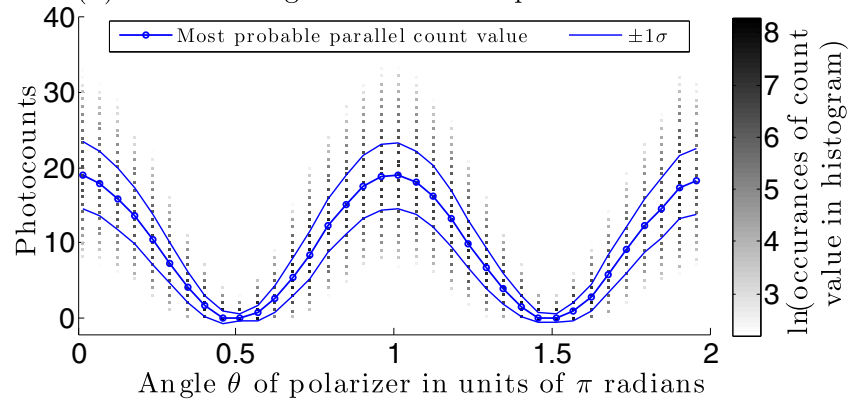

(b) Polarized light calibration: perpendicular channel

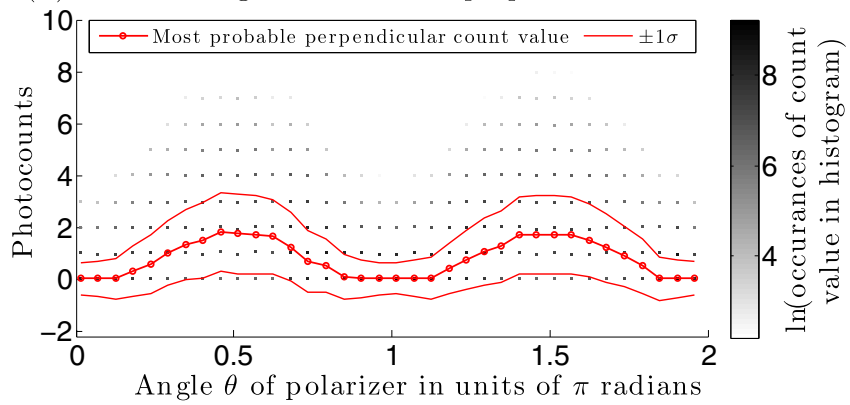

Figure 2. Polarized calibration measurements as a function of incident light polarization angle. Photocounts are given per $1 \mathrm{~min}, 7.5 \mathrm{~m}$ altitude bin. At angle $\theta=0$, the calibration cube polarizer is aligned completely with the parallel measurement channel. At $\theta=0.5 \pi$, it is aligned completely with the perpendicular channel. The grayscale points indicate the extent of scatter in the measurements for each channel. The colour bar indicates the natural logarithm of the number of data points at each location, which is the result of producing a histogram for each angle $\theta$. Data points from all 16000 altitude bins, for all individual minutes of measurement at the angle theta (typically 3 to $5 \mathrm{~min}$ ), are included in the histograms. The truncated distribution of points (because only positive photons may be measured) is evident. Each histogram was fit with a sixth-order polynomial in order to determine the location of the peak photocount rate. This often differed significantly from the mean of the photocount values at that angle and was taken to be the most probable value. The most probable photocount values are given by the blue points (parallel channel; a) and red points (perpendicular channel; b). The thick blue and red lines trace these data points as a visual aid; they are not fit lines. The thin blue and red lines give the $\pm 1 \sigma$ standard deviation of the point distribution at each angle. Note the different $y$-axis limits for each plot; the parallel count rates are far larger than the perpendicular count rates.

larized light which is sent through the rest of the polychromator, including the rotating Polarotor polarizer, and into the PMT.

Omitting the first optics in the detector chain means that this test does not give us a whole-system understanding, although it does allow us to say with certainty whether the downstream optics are contributing any effects other than relative gain (e.g. retardation, rotation) to the signals.
We pose the question, if we consider the optics and detector starting after the focus stage, can we use the simplified Eqs. (3) and (2) to find the calibration constant and then to determine depolarization ratio and depolarization parameter? This is answered in the remainder of Sect. 5. If yes, we can then ask, what is the best estimate for a polarization calibration constant which represents the entire system?, which is addressed in Sect. 6.

\subsection{Matrix description and results of polarized light calibration}

Linearly polarized light is introduced to the receiver. The orientation of the plane of polarization of this light is described as angle $\theta$ with respect to the plane of polarization of the parallel channel. Then measurements are made in the parallel and perpendicular channels. In this scenario, the final Stokes vectors are

$$
\begin{aligned}
& \boldsymbol{I}_{\| \theta}=\frac{G_{\mathrm{PMT}_{\|}}}{2}\left(\begin{array}{cccc}
1 & 1 & 0 & 0 \\
1 & 1 & 0 & 0 \\
0 & 0 & 0 & 0 \\
0 & 0 & 0 & 0
\end{array}\right)\left(\begin{array}{llll}
M_{00} & M_{01} & M_{02} & M_{03} \\
M_{10} & M_{11} & M_{12} & M_{13} \\
M_{20} & M_{21} & M_{22} & M_{23} \\
M_{30} & M_{31} & M_{32} & M_{33}
\end{array}\right) \\
& \frac{G_{\text {cube }}}{2}\left(\begin{array}{cccc}
1 & \cos 2 \theta & \sin 2 \theta & 0 \\
\cos 2 \theta & \cos ^{2} 2 \theta & \frac{1}{2} \sin 4 \theta & 0 \\
\sin 2 \theta & \frac{1}{2} \sin 4 \theta & \sin ^{2} 2 \theta & 0 \\
0 & 0 & 0 & 0
\end{array}\right) G_{\mathrm{gl}}\left(\begin{array}{l}
1 \\
0 \\
0 \\
0
\end{array}\right) I_{\text {lamp }} \\
& \boldsymbol{I}_{\perp_{\theta}}=\frac{G_{\mathrm{PMT}_{\perp}}}{2}\left(\begin{array}{cccc}
1 & -1 & 0 & 0 \\
-1 & 1 & 0 & 0 \\
0 & 0 & 0 & 0 \\
0 & 0 & 0 & 0
\end{array}\right)\left(\begin{array}{llll}
M_{00} & M_{01} & M_{02} & M_{03} \\
M_{10} & M_{11} & M_{12} & M_{13} \\
M_{20} & M_{21} & M_{22} & M_{23} \\
M_{30} & M_{31} & M_{32} & M_{33}
\end{array}\right) \\
& \frac{G_{\text {cube }}}{2}\left(\begin{array}{cccc}
1 & \cos 2 \theta & \sin 2 \theta & 0 \\
\cos 2 \theta & \cos ^{2} 2 \theta & \frac{1}{2} \sin 4 \theta & 0 \\
\sin 2 \theta & \frac{1}{2} \sin 4 \theta & \sin ^{2} 2 \theta & 0 \\
0 & 0 & 0 & 0
\end{array}\right) G_{\mathrm{gl}}\left(\begin{array}{l}
1 \\
0 \\
0 \\
0
\end{array}\right) I_{\text {lamp }}
\end{aligned}
$$

with corresponding signals:

$$
\begin{aligned}
S_{\| \theta}= & G_{\text {cube }} G_{\mathrm{PMT}_{\| \mid}} G_{\mathrm{gl}} \frac{I_{\text {lamp }}}{4} \\
& \left(M_{00}+M_{10}+\left(M_{01}+M_{11}\right) \cos 2 \theta+\left(M_{02}+M_{12}\right) \sin 2 \theta\right) \\
S_{\perp \theta} & =G_{\text {cube }} G_{\mathrm{PMT}_{\perp}} G_{\mathrm{gl}} \frac{I_{\text {lamp }}}{4} \\
& \left(M_{00}-M_{10}+\left(M_{01}-M_{11}\right) \cos 2 \theta+\left(M_{02}-M_{12}\right) \sin 2 \theta\right) .
\end{aligned}
$$

There are very similar equations for the case in which we use backscattered laser light rather than lamp light.

The results of such a test from 5 March 2014 are plotted in Fig. 2. This plot shows the signals in the parallel channel (blue points) and in the perpendicular channel (red points) as a function of cube polarizer angle $\theta$. The cube polarizer was initially placed at an arbitrary angle to ensure that photons were visible in each channel. It was then rotated through a number of steps, spending several minutes at each angle. In total, it was rotated through just more than one full rotation, or $2 \pi$ radians. The absolute angles were determined in post-processing, such that the maximum in the parallel channel is $\theta=0$. All measurements for each angle $\theta$ have been combined for this plot. Photocounts are indicated in units 
Table 2. Parallel and perpendicular signals for angles diagnostic of symmetry in the measurements. Each signal is effectively the same at $0.25 \pi$ and $0.75 \pi$ radians and integer multiples thereof.

\begin{tabular}{lcc}
\hline Angle $\theta(\mathrm{rad})$ & $S_{\|} \pm \sigma_{\|}$ & $S_{\perp} \pm \sigma_{\perp}$ \\
\hline$-0.25 \pi$ & $9.358 \pm 2.5$ & $0.666 \pm 1.3$ \\
$0.25 \pi$ & $9.629 \pm 2.5$ & $0.693 \pm 1.3$ \\
$0.75 \pi$ & $9.269 \pm 2.5$ & $0.701 \pm 1.3$ \\
$1.25 \pi$ & $9.091 \pm 2.5$ & $0.834 \pm 1.3$ \\
$1.75 \pi$ & $9.875 \pm 2.5$ & $0.789 \pm 1.3$ \\
$2.25 \pi$ & $8.919 \pm 2.5$ & $0.830 \pm 1.3$ \\
\hline
\end{tabular}

of "photons per time bin per altitude bin", at a resolution of $1 \mathrm{~min} \times 7.5 \mathrm{~m}$ for each bin. Note the different scales for each panel in Fig. 2: the overall signals in $S_{\|}$far exceed the overall signals $S_{\perp}$. There is approximately a $2^{\circ}$ or 0.035 radian uncertainty in the rotation angles of the calibration cube polarizer when doing this calibration. Freudenthaler (2016) deals at length with the specific issue of uncertainty due to angular error in a calibration optic. Our measurements do not currently have the precision required to warrant a more involved treatment of this uncertainty as they describe.

We could attempt to estimate all seven unknown terms in Eqs. (19) and (20) by allowing them as free parameters in a fit to these signals, but this does not return a unique solution. For CRL, there is a better way to proceed: signal values at some diagnostic angles simplify the equations a great deal by constraining certain calibration constants.

\subsubsection{First constraint: $M_{02}=0$ and $M_{12}=0$}

The signal equations, Eqs. (19) and (20), are simplified a great deal if $M_{02}=0$ and $M_{12}=0$. There is no requirement that the constants $M_{02}$ or $M_{12}$ have these values for given lidar, but a test can verify whether it is the case: $M_{02}$ and $M_{12}$ will both be equal to zero if there is symmetry about $\theta=\pi / 2$ in the curves of both of the signals in Fig. 2, and in particular if the signals at $\theta=\frac{\pi}{4}$ equal those at $\theta=\frac{3 \pi}{4}$ for both the parallel and the perpendicular channel. The results of these measurements are given in Table 2.

For the parallel channel, the signals at these angles are

$$
\begin{aligned}
& S_{|| \theta=\frac{\pi}{4}}=G_{\text {cube }} G_{\mathrm{PMT}_{\|}} G_{\mathrm{gl}} \frac{I_{\text {lamp }}}{4} \\
& \left(M_{00}+M_{10}+\left(M_{02}+M_{12}\right)\right) \\
& S_{|| \theta=\frac{3 \pi}{4}}=G_{\text {cube }} G_{\mathrm{PMT}_{\|}} G_{\mathrm{gl}} \frac{I_{\text {lamp }}}{4} \\
& \left(M_{00}+M_{10}-\left(M_{02}+M_{12}\right)\right) \text {. }
\end{aligned}
$$

If our measurements are symmetric, with $S_{\| \theta=\frac{\pi}{4}}=S_{\| \theta=\frac{3 \pi}{4}}$, then $M_{02}=-M_{12}$.

For the perpendicular channel,

$$
S_{\perp \theta=\frac{\pi}{4}}=G_{\text {cube }} G_{\mathrm{PMT}_{\perp}} G_{\mathrm{gl}} \frac{I_{\mathrm{lamp}}}{4}
$$

$$
\begin{array}{r}
\left(M_{00}-M_{10}+\left(M_{02}-M_{12}\right)\right) \\
S_{\perp \theta=\frac{3 \pi}{4}}=G_{\mathrm{cube} G_{\mathrm{PMT}_{\perp}} G_{\mathrm{gl}} \frac{I_{\text {lamp }}}{4}} \\
\left(M_{00}-M_{10}-\left(M_{02}-M_{12}\right)\right) .
\end{array}
$$

If our measurements are symmetric, with $S_{\perp \theta=\frac{\pi}{4}}=S_{\perp \theta=\frac{3 \pi}{4}}$, then $M_{02}=M_{12}$.

Because the values for $M_{02}$ and $M_{12}$ must not change and are in common for parallel and perpendicular channels, then if both parallel and perpendicular channels are symmetric, both $M_{02}=-M_{12}$ and $M_{02}=M_{12}$ must be true simultaneously, so $M_{02}=-M_{02}$ and $M_{12}=-M_{12}$. Thus, $M_{02}=0$ and $M_{12}=0$.

This simplifies the calibration signal equations to

$$
\begin{aligned}
S_{\| \theta}= & G_{\text {cube }} G_{\mathrm{PMT}_{\|}} G_{\mathrm{gl}} \frac{I_{\text {lamp }}}{4} \\
& \left(M_{00}+M_{10}+\left(M_{01}+M_{11}\right) \cos 2 \theta\right) \\
S_{\perp \theta}= & G_{\mathrm{cube}} G_{\mathrm{PMT}_{\perp}} G_{\mathrm{gl}} \frac{I_{\text {lamp }}}{4} \\
& \left(M_{00}-M_{10}+\left(M_{01}-M_{11}\right) \cos 2 \theta\right) .
\end{aligned}
$$

Each channel's signal values at $\theta=\frac{\pi}{4}$ and $\theta=\frac{3 \pi}{4}$ are equal within their respective uncertainties (Table 2). The mean signal values are $S_{\|}=9 \pm 1$ for parallel and $S_{\perp}=$ $0.8 \pm 0.5$ for perpendicular, with uncertainties calculated using standard error propagation. Therefore, both channels are symmetric about $\theta=\pi / 2$, and the first simplification may be used. Hence, we see that for CRL, $M_{02}=0$ and $M_{12}=0$ are valid for the conditions of this test.

At this point, it is possible to combine Eqs. (25) and (26) into one equation which includes both signals and only the desired calibration constants $\frac{M_{10}}{M_{00}}, \frac{M_{01}}{M_{00}}$, and $\frac{M_{11}}{M_{00}}$, which remain to be determined by a fit to the resulting curve:

$\frac{S_{\| \theta}-S_{\perp \theta}}{S_{\| \theta}+S_{\perp \theta}}=\frac{\frac{M_{10}}{M_{00}}+\frac{M_{11}}{M_{00}} \cos 2 \theta}{1+\frac{M_{01}}{M_{00}} \cos 2 \theta}$.

Again, there is not a unique solution if all three calibration coefficients are free parameters in the fit. It is preferable to see whether the signal measurements indicate any further constraints.

\subsubsection{Second constraint: $M_{10}=M_{01}$ and $M_{00}=M_{11}$}

Further simplifications may be made if the parallel and perpendicular channel signals each go to zero at their respective minima. For parallel, this is at integer multiples of $\theta=\frac{\pi}{2}$, where $\cos 2 \theta=-1$. For perpendicular, this is at $\theta=0$ and at integer multiples of $\theta=\pi$, where $\cos 2 \theta=1$. If this is the case, then

$$
\begin{aligned}
S_{\| \theta}= & 0=G_{\text {cube }} G_{\mathrm{PMT}_{\|}} G_{\mathrm{gl}} \frac{I_{\text {lamp }}}{4} \\
& \left(M_{00}+M_{10}+\left(M_{01}+M_{11}\right)(-1)\right)
\end{aligned}
$$


$0=M_{00}+M_{10}-M_{01}-M_{11}$

and

$$
\begin{gathered}
S_{\perp \theta}=0=G_{\text {cube }} G_{\mathrm{PMT}_{\perp}} G_{\mathrm{gl}} \frac{I_{\text {lamp }}}{4} \\
\left(M_{00}-M_{10}+\left(M_{01}-M_{11}\right)(1)\right) \\
0=M_{00}-M_{10}+M_{01}-M_{11} .
\end{gathered}
$$

In the case that both signals go to zero at their respective locations, Eqs. (28) and (30) are equal, so $M_{10}=M_{01}$ and $M_{00}=M_{11}$.

This leaves the calibration signals as

$$
\begin{aligned}
& S_{\| \theta}=G_{\text {cube }} G_{\mathrm{PMT}_{\|}} G_{\mathrm{gl}} \frac{I_{\text {lamp }}}{4} M_{00}\left(1+\frac{M_{10}}{M_{00}}\right)(1+\cos 2 \theta) \\
& S_{\perp \theta}=G_{\text {cube }} G_{\mathrm{PMT}_{\perp}} G_{\mathrm{gl}} \frac{I_{\text {lamp }}}{4} M_{00}\left(1-\frac{M_{10}}{M_{00}}\right)(1-\cos 2 \theta),
\end{aligned}
$$

in which $\frac{M_{10}}{M_{00}}$ is the only calibration constant needing to be determined. Note that we do not need to know the value of $M_{00}$ or $M_{10}$ individually for calculating depolarization parameter $d$.

For CRL, histograms of the numbers of counts at each angle show that the most probable value for the perpendicular count rate $S_{\perp \theta=0}$ is zero photons per measurement interval. Similar histograms for the parallel channel show that it, too, goes to zero at its minimum (at $\theta=\pi / 2 \mathrm{rad}$ ). Fitted histogram values were used rather than means. The raw lidar photon counting data does not report any values less than zero counts, and noise will artificially increase the total rate. When examining signals larger than zero, the noise takes a Gaussian shape around the mean signal value. For situations in which the true signal is zero, a mean of the measured signal will be reported as a larger value, thus not being indicative of the most probable photon counting result.

As the count rates do indeed go to zero at their respective minima, this second constraint is also appropriate for CRL: $M_{10}=M_{01}$ and $M_{00}=M_{11}$.

\subsection{First result of rotating polarizer test: traditional equations are appropriate for CRL}

The value of $\frac{M_{10}}{M_{00}}$ can now be calculated from the calibration data (see Sect. 5.4), but this is not the most important result from the polarized calibration test. Instead, what matters is that CRL's polychromator optics are acting only as a partial polarizer. $M_{01}=M_{10}$ and $M_{11}=M_{00}$ were identified in Sect. 3.2 as the necessary conditions for which the simpler traditional versions of the depolarization equations Eqs. (3) and (2) are acceptable for CRL. These conditions are fulfilled for CRL. The calibration approach and equations for $d$ and $\delta$ used by others in the remote sensing community is appropriate, despite CRL's many optics between the sky and the analyzing polarizer for the depolarization channel. This assumes that the Dall-Kirkham telescope does not contribute to these quantities in a significant way. This result is reasonable, as the total reflectivity of all telescope mirrors is high for unpolarized light, thus limiting the amount by which light of either polarization plane can be reduced relative to the other. The focus stage of the telescope contains four mirrors in two planes so that polarization induced by two of the mirrors will be cancelled out by that induced in the following two. Thus it is reasonable that the focus stage does not contribute a large amount to $d$ and $\delta$. Nevertheless, the exclusion of the telescope is a limitation of the calibration, and quantities calculated by this test will not be totally representative of the whole lidar system.

\subsection{Determining $\frac{M_{01}}{M_{00}}$ from polarized calibration}

The results from the polarized light calibration may be extended further to calculate a preliminary value of $\frac{M_{10}}{M_{00}}$. Combining Eqs. (31) and (32), we can solve directly for $\frac{M_{10}}{M_{00}}$, the only remaining calibration constant:

$\frac{M_{10}}{M_{00}}=\frac{\cos 2 \theta-\left(\frac{S_{\| \theta}-S_{\perp \theta}}{S_{\| \theta}+S_{\perp \theta}}\right)}{\cos 2 \theta\left(\frac{S_{\| \theta}-S_{\perp \theta}}{S_{\| \theta}+S_{\perp \theta}}\right)-1}$.

In such a calculation, for measurements made at angles $\theta$ where either signal goes to zero, the result for $\frac{M_{10}}{M_{00}}$ is a zero-divided-by-zero fraction. Thus, one must exclude such calibration angles.

This calculation was carried out for the test in Fig. 2. A histogram was made of the calculated $\frac{M_{10}}{M_{00}}$ values, with a peak at $\frac{M_{10}}{M_{00}}=0.77 \pm 0.18$. However, this value has limitations and is not a good representative value for the CRL system: it is not representative of the whole receiver but rather of the polychromator only. It includes no effects of the telescope or focus stage.

Because of these limitations, it is preferable to determine $\frac{M_{10}}{M_{00}}$ as well as $k$, using an unpolarized light test, as demonstrated in the following section (Sect. 6). This determination of the constant employs well-established techniques and has lower uncertainty. Furthermore, it can include all lidar optics. Obtaining a partial-polarizer-like form of the upstream optics Mueller matrix as a result in our polarized calibration test (Sect. 5) allows us to proceed to Sect. 6 with confidence that the tests we will use to determine $k$ and $\frac{M_{10}}{M_{00}}$ for the whole receiver are applicable to the CRL.

\section{Determining $k$ and $\frac{M_{10}}{M_{00}}$ with traditional equations}

The validity of the expressions in Eq. (2) (and therefore Eq. 3 also) has already been demonstrated for CRL in Sect. 5. Therefore these expressions can be used to determine $k$ and/or $\frac{M_{10}}{M_{00}}$ via calibrations in which $d=\delta=1$. This mimics fully depolarized light returning from the sky. Two different methods were used to arrange a $d=\delta=1$ calibration 
setup. The first method forces backscattered lidar light to go through a depolarizing sheet of glassine waxed paper before being measured (Sect. 6.2). The second method involves shining a lamp at the detector through a depolarizing sheet of glassine (Sect. 7). Either of these methods is preferable to using sky light alone without ensuring its total depolarization as it enters the window above the telescope. Even in atmospheric conditions which are thought to be depolarizing (e.g. clouds in which multiple scattering is expected or ice clouds for which complete depolarization of $d=1$ is possible), complete depolarization at all altitudes for the duration of the measurement cannot be ensured.

\subsection{Mueller matrix development of the calibration expressions for $\frac{M_{10}}{M_{00}}$ and $k$}

The matrix equation for the intensity reaching the parallel channel, using the laser as the light source and a perfect depolarizer with attenuation parameter $G_{\mathrm{gl}}$ in front of the receiver is

$$
\begin{aligned}
I_{||}= & \frac{G_{\mathrm{PMT}_{\| \perp}}}{2}\left(\begin{array}{cccc}
1 & 1 & 0 & 0 \\
1 & 1 & 0 & 0 \\
0 & 0 & 0 & 0 \\
0 & 0 & 0 & 0
\end{array}\right) \\
& \left(\begin{array}{cccc}
M_{00} & M_{01} & M_{02} & M_{03} \\
M_{10} & M_{11} & M_{12} & M_{13} \\
M_{20} & M_{21} & M_{22} & M_{23} \\
M_{30} & M_{31} & M_{32} & M_{33}
\end{array}\right) \frac{G_{\mathrm{gl}}}{2}\left(\begin{array}{cccc}
1 & 0 & 0 & 0 \\
0 & 0 & 0 & 0 \\
0 & 0 & 0 & 0 \\
0 & 0 & 0 & 0
\end{array}\right) \\
& b O_{\| \perp \perp}(z)\left(\begin{array}{cccc}
1 & 0 & 0 & 0 \\
0 & 1-d & 0 & 0 \\
0 & 0 & d-1 & 0 \\
0 & 0 & 0 & 2 d-1
\end{array}\right) I_{\text {laser }}\left(\begin{array}{l}
1 \\
1 \\
0 \\
0
\end{array}\right) .
\end{aligned}
$$

Thus, the signals in each channel are

$$
\begin{gathered}
S_{\| d=1}=\frac{G_{\mathrm{PMT}_{\| \perp}} G_{\mathrm{gl}}}{2} b O_{\| \perp}(z) I_{\text {laser }}\left(M_{00}+M_{10}\right) \\
S_{\perp d=1}=\frac{G_{\mathrm{PMT}_{\| \perp}} G_{\mathrm{gl}}}{2} b O_{\| \perp}(z) I_{\text {laser }}\left(M_{00}-M_{10}\right) .
\end{gathered}
$$

The signals from the parallel and perpendicular channel are combined so that we can solve for $\frac{M_{10}}{M_{00}}$ with as many of the unknown factors cancelling out as possible:

$$
\begin{aligned}
& \frac{S_{\| d=1}+S_{\perp d=1}}{S_{\| d=1}}=\frac{2 M_{00}}{M_{00}+M_{10}} \\
& \frac{M_{10}}{M_{00}}=\frac{2}{\frac{S_{\perp d=1}}{S_{\| d=1}}+1}-1 .
\end{aligned}
$$

To calculate $k$, the equation is

$k=\frac{S_{\| d=1}}{S_{\perp d=1}}$.

Note that these equations for $\frac{M_{10}}{M_{00}}$ and $k$ work equally well for the case in which we use a lamp to illuminate the lidar as the several differences in the initial matrix equation cancel out: there is no overlap function and no atmospheric matrix, and we use $I_{\text {lamp }}$ instead of $I_{\text {laser }}$, which also cancels out of the final calibration equations.

The constants $\frac{M_{10}}{M_{00}}$ and $k$ can be calculated from one another. $\frac{M_{10}}{M_{00}}$ tends to be more "forgiving" in terms of accuracy than $k$ does. A small percentage error in $\frac{M_{10}}{M_{00}}$ will yield a larger percent error in $k$. Thus, one must take care when making a selection of which to use despite the ease with which one may convert between them in the absence of estimates of their uncertainties.

\subsection{Physical setup of unpolarized laser calibration to determine $\frac{M_{10}}{M_{00}}$ and $k$}

To include all optics, the depolarized light should be introduced above the roof hatch window. Note that unlike the polarized calibration, we do have access to depolarizing optics which are practical to cover a $1 \mathrm{~m}$ diameter roof hatch window. However, as the lamp is not bright enough when placed in this location, backscattered laser light is better used to test this location.

In this setup, we obtain the depolarized light by running the lidar as usual, with the laser transmitted to the sky and scattered back to the lidar, but we interrupt the optical path of the receiver with a depolarizing sheet (glassine) before the backscattered light enters through the roof window of the detector.

Using a flexible material like glassine was important in the Arctic winter. When the lidar is operating, the glassine sheet is exposed to wind, blowing snow, cold temperatures, and any humidity that may be in the air. Several $41 \mathrm{~cm} \times 51 \mathrm{~cm}$ (16 in. $\times 20$ in.) sheets were taped together with opaque black masking tape to create a larger sheet which could cover the $1 \mathrm{~m}$ diameter lidar roof window. To keep the photon count rates as high as possible during the test, only a single layer of glassine was used, although using two sheets in series ensures more complete depolarization. A $10 \mathrm{~cm}$ diameter circle was removed from the centre of the sheet so that the glassine would not obstruct the laser exit window. Glassine tears easily once its edge is compromised, so it was advantageous to pre-tape any line which was to be cut and then to cut through the taped line. The sheet was placed over the roof window. A foam wedge with a circular hole was snugged down over the glassine sheet around the laser window such that the sheet would be secured and not blow into the laser beam. The four corners of the sheet were held down to the metal surface of the roof around the window, slightly below the level of the window's frame, pulling the glassine very gently taut over the window. Two wooden planks with "feet" on each end provided sufficient tension, and these were wedged into place with foam. 


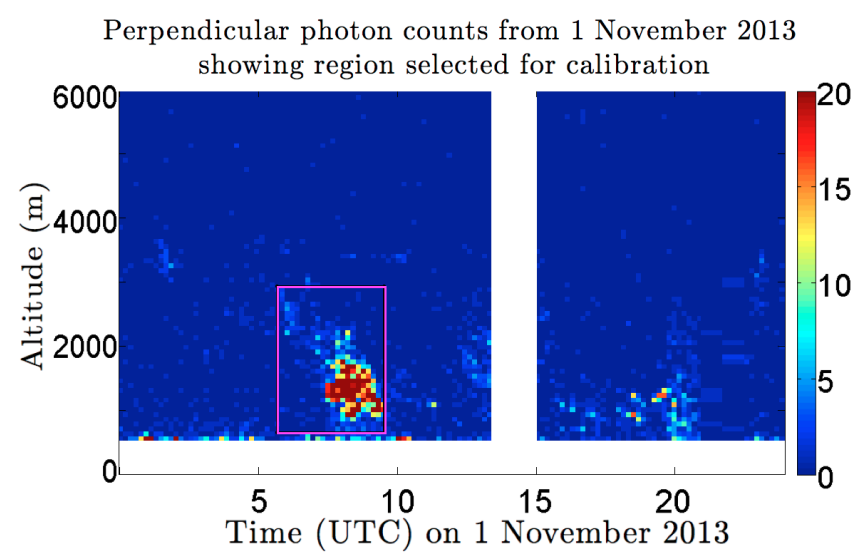

Figure 3. Colour plot of perpendicular channel coadded and corrected photocounts. Magenta box below $3000 \mathrm{~m}$ between 05:00 UTC and 10:00 UTC indicates the region of interest to be used in the calibration analysis.

\subsection{Measurements for unpolarized laser calibration, using example from 1 November 2013}

This test was carried out for over $22.8 \mathrm{~h}$, between 22:40 UTC on 1 November 2013 and 21:00 UTC on 2 November 2013. Figure 3 is a plot of the perpendicular channel backgroundcorrected photocount profiles for the entire calibration measurement, coadded with $10 \times 10$ binning to a resolution of $5 \mathrm{~min} \times 37.5 \mathrm{~m}$. Data from altitudes below $500 \mathrm{~m}$ are routinely rejected from CRL processing because of the differential geometric overlap function of CRL; they are indicated in Fig. 3 in white.

An appropriate time-altitude region of interest must be selected for the calibration. Regions with high-backscatter features, such as clouds, are desirable. The magenta box in Fig. 3 below $3000 \mathrm{~m}$, between 05:00 and 10:00 UTC, shows the region of interest selected for the calibration calculations. This region encompasses a cloud which remained between 1 and $3 \mathrm{~km}$ altitude over the lidar for several hours, as determined by contemporaneous measurements with the Millimetre Cloud Radar and $532 \mathrm{~nm}$ visible Rayleigh elastic CRL lidar channel measurements. Analysis for calibration was attempted on the whole data set as well as just the region of interest which contains the cloud. It was repeated for a variety of coadding times and altitude resolutions. All resolutions provided similar results.

Regions with fewer than one photon per time-altitude bin in either channel and/or signal-to-noise ratios below 0.1 for perpendicular and 1.5 for parallel are rejected from the calculation. Because of the attenuation from the depolarizing sheet, many areas have zero counts in the perpendicular channel, even at low altitudes. Panels (a) and (b) of Fig. 4 plot the parallel and perpendicular photon counts from the region of interest with their respective signal-to-noise ratios plotted to the right in panels (d) and (e).
Once the measurements have been quality controlled, there are several approaches for determining $k$.

1. Calculate a $k$ value individually for each time-altitude bin (Fig. 4c, with associated uncertainty in panel f) and then combine $k$ values into an overall mean constant. This method gives values of $k=20.2 \pm 0.6$. $\frac{M_{10}}{M_{00}}=$ $0.89 \pm 0.05$ is similarly calculated from a mean of individual values of $\frac{S_{\perp}}{S_{\|}}$.

2. Calculate a summed or mean count value for the whole calibration time-altitude space in each of the two channels and then take the ratio of these to calculate one $k$ representative of the whole region. The result using this method is $k=21.0 \pm 0.2$ and $\frac{M_{10}}{M_{00}}=0.910 \pm 0.002$.

The two methods give values which are equal to within their uncertainties. As the uncertainty is smaller using the second approach, this method is selected.

The overall calibration factors for CRL are therefore $\frac{M_{10}}{M_{00}}=0.910 \pm 0.002$ and $k=21.0 \pm 0.2$, calculated using all detector optics. This is quite different from the value of $k=1$, which would be expected for an ideal depolarization lidar in which no upstream optics were interfering with the polarization of returned light.

\section{Determining contributions of individual optics}

To determine which receiver optics contribute most to CRL's high value of $k$, further tests were carried out. These tests determined that the VLWP optic is by far the largest contributor.

\subsection{Physical setup of unpolarized lamp calibration}

A lamp was placed directly upstream of the focus stage, similar to the setup of Sect. 5.1. A depolarizing sheet was installed at various locations within the detector to ensure that all light proceeding from that point was completely unpolarized. This test started with the depolarizing sheet directly before the Polarotor and then moved sequentially upstream, placed between any two optics where there was room to safely insert it (Fig. 1, marked as numbers from 1 through 8). Industrial kitchen grade waxed paper was used for this test, mounted between two frames of foam core to keep it rigid. Measurements were made with the Polarotor in operation as usual. Mean values of parallel and perpendicular counts were used for each calculation of $k$ and $\frac{M_{10}}{M_{00}}$. (Glassine was later found to be more consistently depolarizing from sheet to sheet than are general brands of waxed paper, but we elected not to redo the test in Sect. 7.2, because the particular sheet of waxed paper used here was tested in our laboratory and found to be sufficiently depolarizing.) 

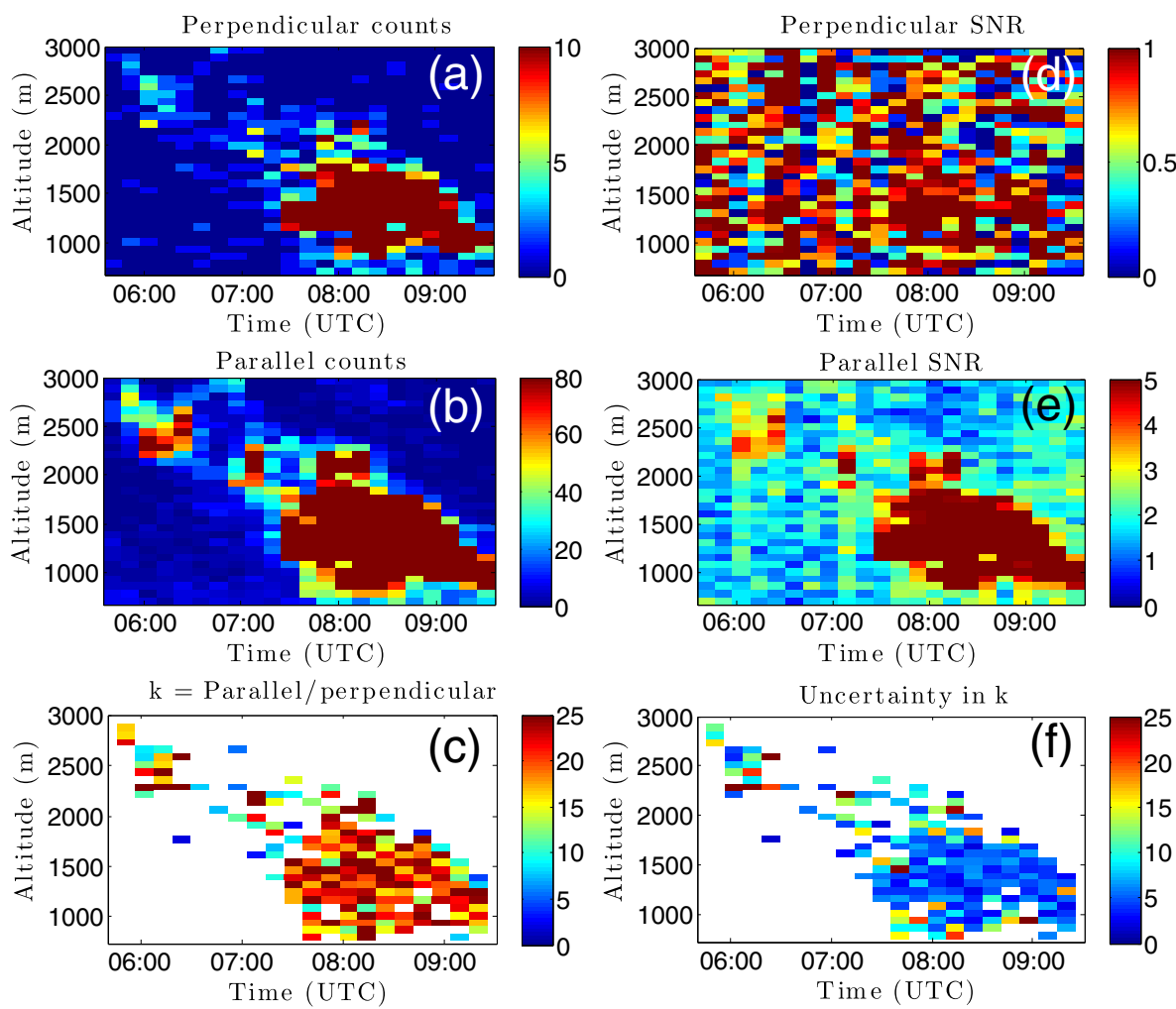

Figure 4. Parallel and perpendicular channel corrected photocounts within the calibration region of interest, with their associated signal-tonoise ratios (SNR) plotted to the right. Bottom left panel shows the ratio of the counts for each time-altitude point $(k)$, with the associated uncertainties in $k$ shown at bottom right.

\subsection{Results: lamp test $\frac{M_{10}}{M_{00}}$ and $k$, setting $d=1$}

The lamp tests were carried out over a $2 \mathrm{~h}$ period on 1 April 2013. The results are listed as tests 1 through 8 in Table 1. The laser calibration test result from Sect. 6.3 is listed as Test 9 in this table, for comparison purposes.

The tests indicate which of our optics are contributing most to the large overall calibration factor of the system. The first test location (Test 1) is as far downstream as it was possible to begin. All subsequent optics (interference filter, focus lens, etc.) are in a closed beam tube, into which it is not feasible to insert a depolarizer. The measurements here showed equal amounts of light in the parallel and perpendicular channels, and $k=1$. This indicates that the collection of optics in this beam tube and the PMT are not contributing to the value of $k=21$ measured for the whole lidar. The " $k$ " described for CRL must therefore include optics in the system and not only the gain of the PMT.

Subsequent tests moving upstream each time indicate that most of the optics in the polychromator are indeed partially polarizing the returned lidar beam, some favouring attenuation of parallel-polarized light (decrease in $k$ and $\left.\frac{M_{10}}{M_{00}}\right)$ and others attenuation of perpendicular-polarized light (increase in $k$ and $\frac{M_{10}}{M_{00}}$ ).
The largest contributor to the overall $k$ value is the VLWP dichroic (compare tests 3 to 5). Its individual contribution is $k_{\mathrm{VLWP}}=6$. Other large contributors are the lenses at the entrance to the polychromator $\left(k_{\text {poly entrance }}=2.2\right.$; compare tests 6 to 7) and the telescope and roof window $\left(k_{\text {telescope }+ \text { window }}=3.12\right.$; compare tests 8 to 9$)$.

The unpolarized lamp tests of Test 7 at the entrance to the polychromator, with $\frac{M_{10}}{M_{00}}=0.719 \pm 0.001$, were made at the same location as the polarized calibration tests of Sect. 5.2, with $\frac{M_{10}}{M_{00}}=0.77 \pm 0.18$. These values are, as expected, equal to within their uncertainties. This provides a link between the two calibration methods.

Tests 7 and 8 took place on either side of the focus stage. Their $\frac{M_{10}}{M_{00}}$ and $k$ results change only by a small amount. Therefore, the focus stage does not contribute significantly to the whole lidar values.

\subsection{Discussion of results from unpolarized lamp tests}

The tests in Sect. 7.2 are important for CRL future planning. First, they indicate that we must calibrate for $k$ by placing the depolarizing optic at the beginning of the optical chain. Many lidar groups (Sassen and Benson, 2001; Alvarez et al., 2006; Wang et al., 2009; Freudenthaler et al., 2009) choose to use calibration lamps, or depolarization optics, part way through 

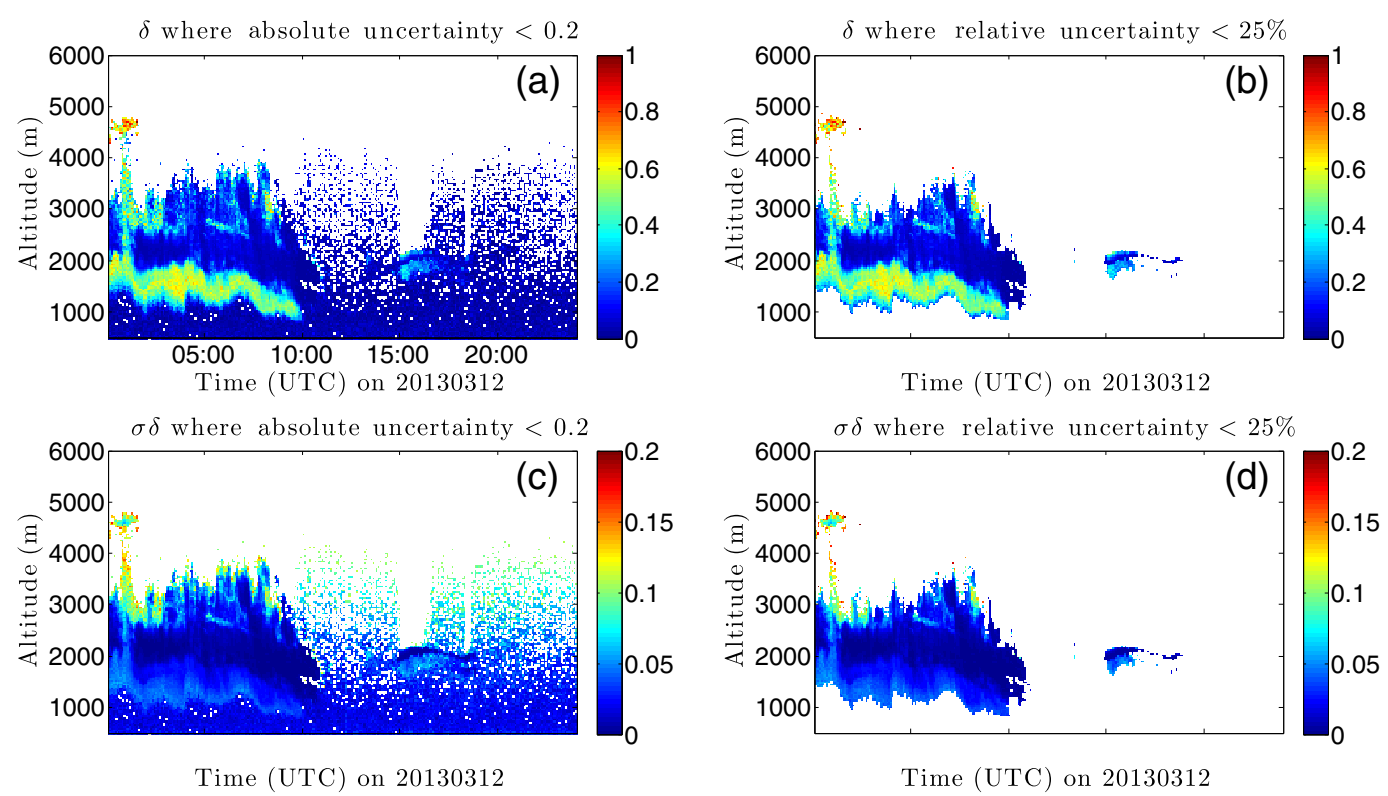

Figure 5. Atmospheric depolarization ratio measurements $(\delta)$ from 12 March 2013, with associated absolute uncertainties $(\sigma \delta)$ in units of depolarization ratio. In the left panels of $\delta(\mathbf{a})$ and $\sigma \delta(\mathbf{c})$, all data points with absolute uncertainty greater than 0.2 have been eliminated. In the right panels of $\delta(\mathbf{b})$ and $\sigma \delta(\mathbf{d})$, points with relative uncertainty greater than $25 \%$ have been eliminated instead. Resolution is 5 min $\times 37.5 \mathrm{~m}$.

their system. An alternative is to use a lamp which scans or is projected over the whole entrance aperture at the first optic of the system. Notably, the lidar group at Howard University uses a mapping lamp applied to a water vapour lidar (Venable et al., 2011). CRL unfortunately does not have the capacity to install such a system at this time. Tests become easier farther downstream in the detector for several reasons: any optic placed at the beginning of the detector chain must be as large as the first optic itself. In the case of CRL, this means a circle with diameter $1 \mathrm{~m}$. Optics of this size are expensive and unwieldy, and are sometimes impossible to obtain. Any optic placed at the beginning of the detector chain will necessarily be outdoors, and will be exposed to the elements. In Eureka this includes temperatures colder than $-50^{\circ} \mathrm{C}$, significant wind, blowing snow, and working on a roof. If a lamp is used for illumination, power is also needed on the roof, which is inconvenient. Going downstream brings the optic inside and makes the required optics smaller. Any optics placed between the telescope and the focus stage must be about $25 \mathrm{~cm}^{2}$. Optics after the focus stage may be as small as $25 \mathrm{~mm}^{2}$. Smaller optics are easier to rotate in a controlled manner (e.g. for polarizer calibrations). At what cost to the calibration and science do these practical advantages come? For CRL, $k$ changes by a factor of 3.4 between the entrance to the polychromator and the entrance to the entire system; the more convenient calibration is insufficient. A second use for these test measurements is that they allow us to see which optics would be most advantageous to change the next time we upgrade optics in the lidar. Naturally, those such as the VLWP filter, which contributes the most to the reduction of the perpendicular signal, would be most advantageous to change.

\section{Sample atmospheric measurements}

Using the best determination of the calibration constant, $k=21.0 \pm 0.2$, (Sect. 6.3), we can determine $d$ and $\delta$ using Eqs. (2) and (3) for a day's measurements to show the performance of CRL. Here we use measurements obtained on 12 March 2013, which we chose because two distinct cloud morphologies are present, as are a variety of signal levels in both depolarization channels, and because some particular places in the plot require special interpretation, which is discussed below. The calibration constant $k$ has been measured to be stable over several years for CRL. Therefore the time between the $k$ calibration example shown in this paper (1 April 2013) and the date of the measurements given in Sect. 8 (12 March 2013) is of no consequence. Figures 5 and 6 show the depolarization ratio, depolarization parameter, and the uncertainties and relative errors for each. Many data points have uncertainties on the order of $10 \%$ and smaller.

\subsection{Uncertainty propagation}

The uncertainty and errors were propagated using standard uncertainty propagation rules through low-level data processing equations and then through the algebraic functions shown in this document, assuming uncorrelated errors (for a full account see McCullough, 2015). The Licel system uses simultaneous photon counting and analogue detection for the 
PMTs, and thus two signals are merged together for each "measurement channel". In all cases, the data are read into software and overflow flagged bits are removed. Uncertainties are initially propagated separately for photon counting and analogue signals. Then they are combined.

For photon counting data, most of the uncertainty in the photon counting channel is derived from shot noise. This uncertainty is then propagated through the equations of the low level data processing in the following order: (1) begin with photon shot noise. This is applicable for both raw counts and raw background counts. This is a statistical uncertainty. (2) Propagate this uncertainty through the dead time equation, in which the dead time uncertainty is a systematic uncertainty (cannot improve by coadding or longer integration). (3) Propagate the uncertainty through the coadding equations. This generally reduces the overall size of the relative uncertainty (see Sect. 8.1) in the measurement. (4) Determine the uncertainty in the background level, which was determined from the dead time corrected background values at high altitudes. This is a statistical uncertainty, because it includes the shot noise uncertainty. (5) Add the dead-time corrected, coadded shot uncertainty and the dead-time corrected, coadded background uncertainty in quadrature to get a total photon counting uncertainty for each data point.

For analogue data, the procedure is different: (1) begin with raw analogue digital signals. (2) Account for the analogue-to-digital converter uncertainty. (3) Include the analogue shot noise uncertainty (a statistical uncertainty). (4) Account for the uncertainty in turning analogue count rates into range-scaled values. (5) Remove dark count profiles and account for this uncertainty. (6) Determine the uncertainty in the sky background constant determined from background values at high altitudes. This is a statistical uncertainty, because it includes the shot noise uncertainty. Then account for it during background subtraction. (7) Account for the uncertainty involved in converting analogue rangescaled voltage signals to equivalent photon count rates (uncertainty depends on precision when calculating the "gluing coefficients" or "merging coefficients" based on fits to lidar data).

Finally, the two profiles, photon counting and analogue, are combined into a single resulting photocount profile for each measurement channel, for each (coadded) time bin. At each point, the raw photon counting rate determines which contributor's count value will be included: photon counting or analogue. The uncertainty at each point in the combined profile is then simply the uncertainty of the contributing data point. These combined profiles can then be used for higher analysis into useful data products such as depolarization ratio and depolarization parameter. The uncertainties in these profiles are the uncertainties on the values $S_{\|}$and $S_{\perp}$, which appear in Eqs. (2) and (3) of this paper. We then propagate these uncertainties through the equations Eq. (2) and (3), again assuming uncorrelated errors, to arrive at an overall estimate for the absolute uncertainty in $\delta$ and $d$.
The uncertainties given as "absolute uncertainty" are the $1 \sigma$ combined effects of systematic and statistical uncertainties and errors and are expressed as, for example, $d=$ $0.200 \pm 0.003$, in which the \pm uncertainty is given in the same units as $d$ and would directly give the size of an error bar on a 2-D plot. Some confusion may arise when comparing values of $\delta$ and $d$ from this document to those given elsewhere. Because depolarization parameters can exist from values of 0 to 1 , some authors express " $d=0.2$ " as " $d=20 \%$ ", for example. This is never done in the present document, precisely to avoid ambiguity with the relative errors discussed next. All values of $\delta$ and $d$ are given as decimal, non-percent numbers, as are absolute uncertainty values. In other words, expressed with absolute uncertainty, we have $x \pm \sigma_{x}$.

Relative uncertainty aims to describe the uncertainty in relation to the measured size of the value of $d$, and is expressed as a percent: $d=0.200 \pm(0.003 / 0.2) \times 100 \%$, which is $d=0.2 \pm 1.5 \%$, for the same example. In other words, expressed as relative uncertainty, we have $x \pm\left(\sigma_{x} / x\right)$.

\subsection{Interpretation of sample atmospheric measurements}

Just below $2 \mathrm{~km}$ altitude, a region of high depolarization is evident with low uncertainty. This implies that this region of the cloud is icy rather than made of liquid droplets. As altitude increases in the cloud, the depolarization drops. This could be because the cloud has suddenly turned into liquid droplets, but there are other factors to consider. First, the uncertainty is higher in these regions, but this does not tell the whole story. This calculated uncertainty expresses only the uncertainty in the calculated result from Eq. (3) based on the number of photons returned in each measurement channel and the statistical uncertainty in each of these measurements. As Eq. (3) is not applicable in circumstances of multiple scattering, any value calculated using this equation for time-altitude locations experiencing such scattering will not be valid as a proxy for particle phase - despite our (possibly precise) ability to calculate it. In the example presented here, the assumption of no multiple scattering is decreasingly trustworthy high in the cloud, as photons have to pass through the thick cloud below twice. In future, it would be advantageous to quantify the added uncertainty due to the likelihood of multiple scatters. The overall effect of multiple scattering is an increase in depolarization, but detailed effects are expected to differ for liquid and ice particle cases. According to laboratory experiments and theoretical calculations, multiple scattering from liquid droplets is likely to induce depolarization of no more than 3 to $4 \%$ for a lidar with a field of view of $1 \mathrm{mrad}$, with depolarization depending also on particle number density (Liou, 1972; Liou and Lahore, 1974). Atmospheric measurements of water clouds support these estimates and demonstrate a linear increase in multiple-scattering-induced depolarization with increased 


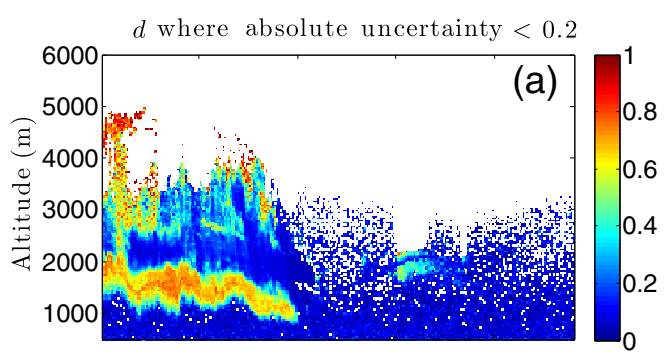

Time (UTC) on 20130312

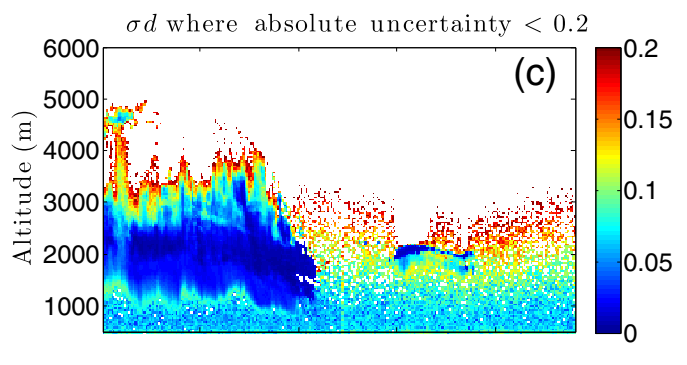

Time (UTC) on 20130312

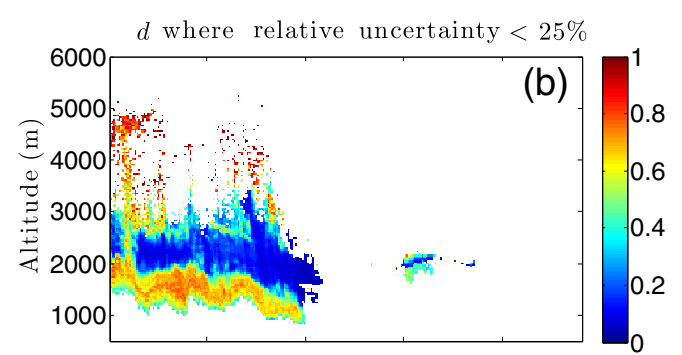

Time (UTC) on 20130312

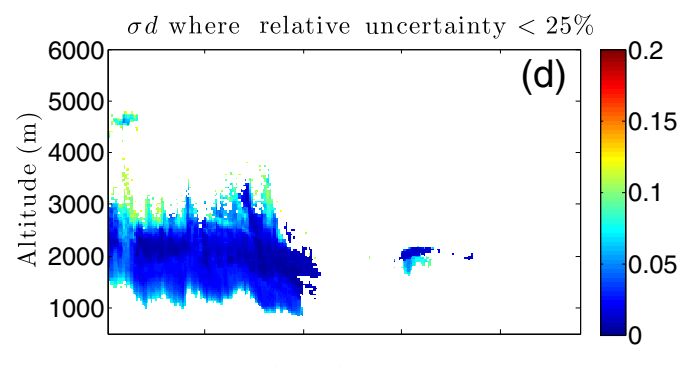

Time (UTC) on 20130312

Figure 6. Atmospheric depolarization parameter measurements from 12 March 2013, with associated absolute uncertainties in units of depolarization parameter. In the left panels of $d$ (a) and $\sigma d$ (c), all data points with absolute uncertainty greater than 0.2 have been eliminated. In the right panels of $d$ (b) and $\sigma d$ (d), points with relative uncertainty greater than $25 \%$ have been eliminated instead. Resolution is $5 \mathrm{~min} \times 37.5 \mathrm{~m}$.

receiver acceptance angle (Sassen and Petrilla, 1986). More recent Monte Carlo simulations of water clouds find that a third-order polynomial describes the relation between depolarization ratio and multiple-scattering fraction ( $\mathrm{Hu}$ et al., 2006). Ray tracing code for ice particles also indicates that multiple scattering leads to an increase in the depolarization ratio, but the magnitude of the increase showed a strong dependence on cloud optical thickness and particle shape (Noel et al., 2002). A full integration of these effects is beyond the scope of the present paper. Therefore care must be taken when interpreting the depolarization values presented herein, even those with low measurement uncertainty.

A further possible contributing factor is that the two channels may have differing amounts of extinction when the transmission function of the atmosphere is polarization dependent. The most trustworthy depolarization values are those for which we have both low uncertainty and reasonable confidence that we have a low extinction single-scattering situation for every photon involved in the data for that timealtitude bin. Examples of such locations in this example night are the lower portions of the large cloud before 10:00 UTC and, to a smaller degree, the entire small cloud between 15:00 and 20:00 UTC.

It may be most sensible to cut out regions which are not providing trustworthy depolarization values based on criteria discussed above. The values could be cut based either on relative error or on absolute error. The latter is chosen in this situation because a measurement of a depolarization parameter $d=0.01 \pm 0.01$ is still meaningful, despite hav- ing $100 \%$ relative error. Interpretations deal with cutoffs between different values of $d$ and the measurements need to be sensitive enough to discern this cutoff, without eliminating an excessive number of measurements. Compare the data coverage of the upper left panel (a) in either Fig. 6 or Fig. 5 with the coverage of its corresponding upper right panel (b). Far more low-depolarization values are kept by cutting off using the absolute uncertainty rather than the relative uncertainty, without losing any interpretation confidence. Extra information is available at $3 \mathrm{~km}$ just after 05:00 UTC: there are distinct regions of increased depolarization parameter which are retained when cutting depolarization parameter on absolute uncertainty rather than on relative uncertainty. Although these are above thick cloud, and so multiple scattering may influence the interpretation of the specific values of $d$, the relative values can still be instructive, so it is useful to retain these. Features such as liquid layers within otherwise frozen clouds, or frozen parts within liquid clouds, would be detectable in similar situations, and regions of aerosols within clear air as well. Depending on the application, it may be of use to some lidar users to know simply that the cloud is inhomogeneous in cloud particle phase or that an aerosol layer is present, without the specific microphysical details which are usually available with a well-constrained absolute depolarization parameter value.

Further coadding to lower resolution would help with the data coverage by increasing the number of perpendicular photocounts per bin. On some dates, measurements can be retained in this way up to 8 to $10 \mathrm{~km}$ altitude. However, this 
can only be carried out to a certain point, after which the lowresolution depolarization measurements will be misleading. For example, any instances of thin liquid layers (low $d$ and $\delta$ ) residing within an ice cloud (high $d$ and $\delta$ ) would, at low resolution, show a smooth region with intermediate values of $d$ and $\delta$. As such intermediate values of $d$ and $\delta$ are not actually present anywhere within the binned region, it would be incorrect to use these values for interpretation of the cloud itself. For this reason, measurements are kept at as high a resolution as possible, while still retaining as many measurement points as possible.

\section{Future work}

CRL can now make depolarization parameter measurements at a precision of $<10 \%$ relative uncertainty in clouds at a resolution of $5 \mathrm{~min} \times 37.5 \mathrm{~m}$ (Sect. 8) despite the less than optimum optical configuration of CRL. The major difficulty for CRL is in receiving sufficient perpendicular signal at the depolarization PMT, as indicated by the very large calibration value of $k=21$ found in the system, and by the low photocount rates in the perpendicular channel during atmospheric measurements. For any unpolarized light which scatters back to the roof window, the portion which would be allowed through the perpendicular analyzer near the PMT is preferentially suppressed by lidar receiver optics in comparison to the portion of unpolarized light which would be allowed through the parallel analyzer. There are several possibilities for improvement of the depolarization measurements: changes to the depolarization parameter calculation method and changes to lidar hardware. Suggestions for each of these are provided in Sects. 9.1 and 9.2 respectively.

\subsection{New analysis method}

We have developed a new depolarization calculation method by extending the Mueller matrix instrument characterization method shown in this paper to a third CRL measurement channel: the unpolarized $532 \mathrm{~nm}$ Rayleigh elastic channel. This method will be discussed in detail in a companion paper (McCullough et al., 2017) and is available in McCullough (2015). In the new method, low-resolution traditional $d$ values, as calculated in the present paper, are used to create a nightly calibration profile. The improved calculation method produces depolarization parameters with similar uncertainties as the traditional method, but at much higher resolutions of $2 \mathrm{~min} \times 15 \mathrm{~m}$ and with far fewer data points lost to low signals, and therefore better measurement coverage of the atmosphere above the lidar. Alternately, the results can be expressed at similar resolution to the traditional depolarization products for CRL but with much higher precision.

\subsection{Hardware upgrades}

Hardware upgrades are the other option for improving the CRL depolarization measurements. Any or all of these would improve both calculation methods for $d$ by increasing signals in the perpendicular channel. In the context of the calibrations in this paper, hardware improvements seek to reduce the value of $k$ by increasing the number of perpendicular photons which reach the depolarization PMT. These hardware improvements are also relevant for any other lidar which does not have access to a polarization-independent channel at the same wavelength as its depolarization channels and thus cannot take advantage of the new calculation method from Sect. 9.1.

Specifically, any of the following hardware changes would improve signals in the perpendicular channel. First, the laser's polarization could be rotated by $90^{\circ}$, allowing the collection optics in the polychromator to suppress the large parallel signal while enhancing the small perpendicular signal. Second, putting a quarter-waveplate at the entrance to the polychromator would also help balance the signals for the same reason stated above. Third, replacing the VLWP filter with one which is less polarizing, or less polarizing in the perpendicular-suppressing direction, would reject as few as possible the perpendicular photons which enter the telescope. Fourth, using two depolarization PMTs would allow for different gain settings individually optimized for the parallel and perpendicular channels, but this change to the configuration is not possible with the current electronics. CRL's setup uses a single PMT to make both parallel and perpendicular measurements, and there is no switching of PMT gain settings between laser shots. Therefore, the gain setting must be a compromise of what is best for the high-signal parallel measurements and the low-signal perpendicular measurements. In practice means a lower gain setting is required to optimize the parallel channel to avoid PMT saturation. The high dynamic range of the combined analogue and photon counting of the Licel recorders helps somewhat, but having intensities of more comparable levels to begin with would be a better choice. Fifth, moving the depolarization PMT further upstream in the detector, perhaps with a pick-off beam splitter at the beginning of the optical chain, would remove many of the partially polarizing optics from the path to the depolarization PMT, thus rejecting fewer of the perpendicular photons than is currently the case. Any of the CRL hardware improvements here described would improve depolarization measurements made using the traditional equations for CRL or for any similar lidar and would also improve measurements made using the newly developed three-channel calculation technique.

Throughout this work, $k$ values far from unity have been presented as being undesirable. There can, however, be advantages to a setup with $k \neq 1$, albeit with $k<1$ rather than $k>1$ as CRL has. Consider that the scattering properties of the atmosphere lead to maximum $d$ and $\delta$ values of 1 for total 
depolarization and 0 for no depolarization. For $d=1$, half of the backscattered intensity reaching the roof window would be admitted to an ideal $(k=1)$ parallel channel, and the other half of the intensity would be admitted to the perpendicular channel. For $d=0$, all of the intensity is parallel, and none is perpendicular. Therefore, the maximum perpendicular intensity ever possible to be backscattered to the lidar's roof window and through the receiver is only half the maximum parallel signal ever possible. With a whole-system calibration value $k=1$, the maximum measured perpendicular signal will also be half of the maximum measured parallel signal. Values of $k>1$ suppress the perpendicular signal even further. Therefore, $k>1$ values for CRL are leading to the preferential attenuation of already-smaller signals. If CRL instead had $k$ values just as far from unity, but with $k<1$, the larger parallel signal would instead be preferentially attenuated. This analysis leads to the interesting conclusion that for CRL, $k<1$ optics would lead to better signal-to-noise ratios overall and lower uncertainty in products derived from ratios of the two signals.

The new analysis technique makes it unlikely that the physical changes to CRL will be carried out in the near future, as they are less critical to the success of the CRL depolarization measurements than they would be if the traditional method were the only calculation possibility available. Therefore, all efforts have been directed toward the validation of the new analysis method.

\section{Conclusions}

Depolarization measurement capability has been added to the CRL lidar at Eureka, Nunavut, in the Canadian High Arctic. A single Polarotor rotating Glan-Thomson prism, focusing lens, interference filter, and PMT were installed to make parallel and perpendicular measurements on alternate laser shots. Most of CRL's received $532 \mathrm{~nm}$ light is directed by a VLWP filter into the pre-existing visible Rayleigh elastic channel. A pellicle beam splitter was installed downstream of this optic to direct a small portion of the remaining $532 \mathrm{~nm}$ light into the new depolarization measurement channel. Characterization tests have demonstrated that the traditional depolarization ratio and depolarization parameter equations and their calibration methods are appropriate to be used for CRL, despite the lidar not having been built specifically to make polarization measurements.

A Polarotor start delay calibration defined the parallel and perpendicular measurement angles for the depolarization channels. Uncertainty induced by this calibration is negligible compared to other sources of uncertainty in the CRL's measurements of $\delta$ and $d$, with photon counting noise being by far the dominant source of uncertainty in the measurements. Further calibrations characterize the response of the depolarization detection channels.
A calibration which introduces polarized lamp light at a variety of angles into the detector at the location immediately downstream of the lidar's focus stage allowed a single Mueller matrix to describe the collective effects of all receiver optical elements between the telescope's focus stage and the Polarotor polarizing beam splitter. The following matrix elements, and ratios of matrix elements, were measured using this polarized lamp test: $M_{02}=0 ; M_{12}=0 ; M_{11}=$ $M_{00} ; M_{10}=M_{10} ; \frac{M_{10}}{M_{00}}=0.77 \pm 0.18$. Because $M_{11}=M_{00}$ and $M_{10}=M_{01}$, we conclude that the optics represented by the "upstream optics" matrix act as a partial polarizer (without rotation, retardation, or other optical effects) on the calculation of $d$ and $\delta$. Therefore, the traditional calculation equations for $d$ (Eq. 2) and $\delta$ (Eq. 3), which use the single calibration constant $\frac{M_{10}}{M_{00}}$ and $k$ respectively, are valid for the CRL. Practical limitations of this test mean that the roof window, telescope, and focus stage optics are excluded from evaluation.

Values for $\frac{M_{10}}{M_{00}}$ and $k$ representative of the whole receiver were determined by the next calibration, which was done with the lidar's laser firing to the sky. A glassine depolarizing sheet was placed upstream of the roof window to depolarize the backscattered lidar returns. This calibration includes all lidar receiver optics. It assumes that the roof window, telescope, and focus stage optics are not contributing effects other than those of partial polarizers but allows them to preferentially attenuate light according to its polarization. The results of this test are $\frac{M_{10}}{M_{00}}=0.910 \pm 0.002$ and $k=21.0 \pm 0.2$. These values are representative of the entire lidar receiver, so are applied to CRL's routine atmospheric measurements of depolarization.

To investigate the relative contributions of lidar receiver optics to the total values of $\frac{M_{10}}{M_{00}}$ and $k$, a final calibration was carried out. Unpolarized lamp light was introduced into the lidar's receiver at numerous locations, and $\frac{M_{10}}{M_{00}}$ and $k$ were calculated for each subset of optics. These detailed optic-byoptic measurements of $\frac{M_{10}}{M_{00}}$ and $k$ indicate that the VLWP filter is the most highly polarizing optic, increasing $k$ by a factor of 5.6. The next most polarizing optic is the combination of the telescope and roof window, which increase $k$ by a factor of 3.1. Collimating optics at the entrance of the polychromator increase $k$ by a factor of 2.2, and the collimating lens directly upstream of the pellicle beam splitter increases $k$ by a factor of 1.1. The focus stage's arrangement of four mirrors in two planes has a small effect, increasing $k$ by a factor of 1.1. The pellicle beam splitter itself and the UV long-wave pass optic both decrease $k$, with factors of 0.6 and 0.78 respectively. The focusing lens and interference filter downstream of the Polarotor contribute no change to $k$.

The polarized and unpolarized calibration tests have one test location in common. The unpolarized lamp light test upstream of the focus stage gives results of $\frac{M_{10}}{M_{00}}=0.719 \pm 0.001$ and $k=6.12 \pm 0.02$, which are within uncertainty to the values calculated from that location during the polarized-light calibration test. 
The whole-system representative $\frac{M_{10}}{M_{00}}=0.910 \pm 0.002$ and $k=21.0 \pm 0.2$ results are applied to a 24 -hour long atmospheric measurement. This demonstrates the depolarization ratio and depolarization parameter for the sky above Eureka on 12 March 2013, at a resolution of $5 \mathrm{~min} \times 37.5 \mathrm{~m}$. During this period, a thick, partially frozen cloud was present before 10:00 UTC, and the reduced reliability of the depolarization measurements farther into the thick cloud is evident as multiple scattering becomes important. A non-frozen cloud with regions of higher depolarization material above it was present after 15:00 UTC. CRL was also found to be sensitive to the low depolarization regions of clear air below the 15:00 UTC cloud.

Typical depolarization parameter absolute uncertainties at $\mathrm{CRL}$ are on the order of \pm 0.05 ( $\leq 10 \%$ relative uncertainty) within clouds at time and altitude resolutions of $5 \mathrm{~min}$ and $37.5 \mathrm{~m}$ respectively, with higher precision and higher resolution possible in select cases, and uncertainty somewhat larger $( \pm 0.1)$ at the same altitude outside of clouds.

This work has resulted in a well-characterized depolarization measurement system at CRL, which had no prior depolarization measurement capability. Using a similar Mueller algebra exercise, these calibration methods may be applied to other lidars to elucidate the properties of the optics. This work shows that it is possible to add depolarization capability to lidars which were not originally designed specifically for polarization measurements.

Data availability. Data used in this paper are available upon request from corresponding author (emccull2@uwo.ca).

Author contributions. EM McC was responsible for the installation and calibration of depolarization hardware, data analysis and development of method, writing of analysis MATLAB code, and manuscript preparation. This work formed part of EM McC's doctoral thesis. RJS was responsible for the supervision of the doctoral thesis and contribution to manuscript preparation. JRD was the principal investigator of PEARL laboratory and contributed to manuscript preparation. TJD was the principal investigator of CRL lidar at the time of this work and responsible for development of the CRL laboratory. GJN was responsible for the original design of depolarization channel for CRL; he provided instruction in installation and initial data processing in Python, as well as extensive discussions regarding calibration and analysis, and contributed to manuscript preparation. JH was responsible for maintenance and testing of depolarization hardware, as well as lidar operations. CPT was responsible for lidar operations, provided assistance in the laboratory, and contributed to manuscript preparation. $\mathrm{CP}$ provided instruction on lidar operations. JD provided instruction on lidar operations. KAW was principal investigator of polar sunrise ACE/OSIRIS Arctic validation campaigns of which these CRL measurements are one component.
Competing interests. The authors declare that they have no conflict of interest.

Acknowledgements. PEARL has been supported by a large number of agencies, whose support is gratefully acknowledged: the Canadian Foundation for Innovation, the Ontario Innovation Trust, the (Ontario) Ministry of Research and Innovation, the Nova Scotia Research and Innovation Trust, the Natural Sciences and Engineering Research Council, the Canadian Foundation for Climate and Atmospheric Science, Environment and Climate Change Canada, Polar Continental Shelf Project, the Department of Indigenous and Northern Affairs Canada, and the Canadian Space Agency. This work was carried out during the Canadian Arctic ACE/OSIRIS Validation Campaigns of 2010, 2011, 2012, and 2013, which are funded by the Canadian Space Agency, Environment and Climate Change Canada, the Natural Sciences and Engineering Research Council of Canada, and the Northern Scientific Training Program. This particular project has also been supported by NSERC Discovery Grants and Northern Supplement Grants held by James R. Drummond, Robert J. Sica, and Kaley A. Walker and the NSERC CREATE Training Program in Arctic Atmospheric Science (PI: Kim Strong). In addition, the authors thank the following groups and individuals for their support during field campaigns at Eureka: PEARL site manager Pierre Fogal; Canadian Network for the Detection of Atmospheric Change (CANDAC) operators: Mike Maurice, Peter McGovern, Alexei Khmel, Paul Leowen, Ashley Harret, Keith MacQuarrie, Oleg Mikhailov, and Matt Okraszewski; and the Eureka Weather Station staff.

Edited by: Ulla Wandinger

Reviewed by: three anonymous referees

\section{References}

Adachi, H., Shibata, T., Iwasaka, Y., and Fujiwara, M.: Calibration method for the lidar-observed stratospheric depolarization ratio in the presence of liquid aerosol particles, Appl. Optics, 40, 6587-6595, 2001.

Alvarez, J. M., Vaughan, M. A., Hostetler, C. A., Hunt, W. H., and Winker, D. M.: Calibration Technique for Polarization-Sensitive Lidars, American Meteorological Society, 23, 683-699, 2006.

Andover Corporation: 500-599 nm standard bandpass optical filters technical specifications, 2015.

Baibakov, K., O’Neill, N. T., Ivanescu, L., Duck, T. J., Perro, C., Herber, A., Schulz, K.-H., and Schrems, O.: Synchronous polar winter starphotometry and lidar measurements at a High Arctic station, Atmos. Meas. Tech., 8, 3789-3809, https://doi.org/10.5194/amt-8-3789-2015, 2015.

Biele, J., Beyerle, G., and Baumgarten, G.: Polarization lidar: Corrections of instrumental effects, Opt. Express, 7, 427-435, 2000.

Boer, G. D., Eloranta, E., and Shupe, M.: Arctic mixed-phase stratiform cloud properties from multiple years of surface-based measurements at two high-latitude locations, J. Atmos. Sci., 66, 2874-2887, 2009.

Bourdages, L., Duck, T. J., Lesins, G., Drummond, J. R., and Eloranta, E. W.: Physical properties of High Arctic tropospheric 
particles during winter, Atmos. Chem. Phys., 9, 6881-6897, https://doi.org/10.5194/acp-9-6881-2009, 2009.

Bravo-Aranda, J. A., Navas-Guzmán, F., Guerrero-Rascado, J. L., Pérez-Ramírez, D., Granados-Muñoz, M. J., and AladosArboledas, L.: Analysis of lidar depolarization calibration procedure and application to the atmospheric aerosol characterization, Int. J. Remote Sens., 34, 3543-3560, 2013.

Bravo-Aranda, J. A., Belegante, L., Freudenthaler, V., AladosArboledas, L., Nicolae, D., Granados-Muñoz, M. J., GuerreroRascado, J. L., Amodeo, A., D’Amico, G., Engelmann, R., Pappalardo, G., Kokkalis, P., Mamouri, R., Papayannis, A., NavasGuzmán, F., Olmo, F. J., Wandinger, U., Amato, F., and Haeffelin, M.: Assessment of lidar depolarization uncertainty by means of a polarimetric lidar simulator, Atmos. Meas. Tech., 9, 49354953, https://doi.org/10.5194/amt-9-4935-2016, 2016.

Bu, L., Sa, R., and Kim, D.: Calibration method of polarization lidar based on Jones matrix, Optik-International Journal for Light and Electron Optics, 130, 834-839, 2017.

Bush, V.: Summary Technical Report of Division 16, NDRC, vol. 3, Non-Image Forming Infrared, Tech. rep., The National Defense Research Committee, 1946.

Cairo, F., Di Donfrancesco, G., Adriani, A., Pulvirenti, L., and Fierli, F.: Comparison of various linear depolarization parameters measured by lidar, Appl. Optics, 38, 4425-4432, 1999.

Curry, J., Rossow, W., Randall, D., and Schramm, J.: Overview of Arctic cloud and radiation characteristics, J. Climate, 9, 17311764, 1996.

David, G., Miffre, A., Thomas, B., and Rairoux, P.: Sensitive and accurate dual-wavelength UV-VIS polarization detector for optical remote sensing of tropospheric aerosols, Appl. Phys. B-Lasers O., 108, 197-216, 2012.

Di, H., Hua, H., Cui, Y., Hua, D., Li, B., and Song, Y.: Correction technology of a polarization lidar with a complex optical system, J. Opt. Soc. Am. A, 33, 1488-1494, 2016.

Doyle, J. G., Lesins, G., Thackray, C. P., Perro, C., Nott, G. J., Duck, T. J., Damoah, R., and Drummond, J. R.: Water vapor intrusions into the High Arctic during winter, Geophys. Res. Lett., 38, 1-5, 2011.

Eloranta, E. W. and Piironen, P.: Depolarization measurements with the high spectral resolution lidar, in: Proceedings of the 17th ILRC, Sendai, Japan, vol. 7574, 1994.

Flynn, C. J., Mendoza, A., Zheng, Y., and Mathur, S.: Novel polarization-sensitive micropulse lidar measurement technique, Opt. Express, 15, 2785-2790, 2008.

Freudenthaler, V.: About the effects of polarising optics on lidar signals and the $\Delta 90$ calibration, Atmos. Meas. Tech., 9, 41814255, https://doi.org/10.5194/amt-9-4181-2016, 2016.

Freudenthaler, V., Esselborn, M., Wiegner, M., Heese, B., Tesche, M., Ansmann, A., MüLLER, D., Althausen, D., Wirth, M., Fix, A., Ehret, G., Knippertz, P., Toledano, C., Gasteiger, J., Garhammer, M., and Seefeldner, M.: Depolarization ratio profiling at several wavelengths in pure Saharan dust during SAMUM 2006, Tellus B, 61, 165-179, 2009.

Gimmestad, G. G.: Reexamination of depolarization in lidar measurements, Appl. Optics, 47, 3795-3802, 2008.

Hayman, M. and Thayer, J. P.: Explicit description of polarization coupling in lidar applications, Opt. Lett., 34, 611-613, 2009.

Hayman, M. and Thayer, J. P.: General description of polarization in lidar using Stokes vectors and polar decomposition of Mueller matrices vectors and polar decomposition of Mueller matrices, J. Opt. Soc. Am., 29, 400-409, 2012.

Hohn, D. H.: Depolarization of a laser beam at 6328 Angstroms due to the atmospheric transmission, Appl. Optics, 8, 367-370, 1969.

Hu, Y., Liu, Z., Winker, D., Vaughan, M., Noel, V., Bissonnette, L., Roy, G., and McGill, M.: Simple relation between lidar multiple scattering and depolarization for water clouds, Opt. Lett., 31, 1809-1811, 2006.

Intieri, J. M., Shupe, M. D., Uttal, T., and McCarty, B. J.: An annual cycle of Arctic cloud characteristics observed by radar and lidar at SHEBA, J. Geophys. Res., 107, SHE 5-1-SHE 5-15, 2002.

Kalesse, H., de Boer, G., Solomon, A., Oue, M., Ahlgrimm, M., Zhang, D., Shupe, M. D., Luke, E., and Protat, A.: Understanding Rapid Changes in Phase Partitioning between Cloud Liquid and Ice in Stratiform Mixed-Phase Clouds: An Arctic Case Study, Mon. Weather Rev., 144, 4805-4826, 2016.

Kaul, B. V., Samokhvalov, I. V., and Volkov, S. N.: Investigating particle orientation in cirrus clouds by measuring backscattering phase matrices with lidar, Appl. Optics, 43, 6620-6628, 2004.

Licel GmbH: Rotating Polarizer Multispectral polarization measurements, Tech. rep., Licel lidar computing and electronics, 2006.

Liou, K. and Lahore, H.: Laser sensing of cloud composition: a backscattered depolarization technique, J. Appl. Meteorol., 13, 257-263, 1974.

Liou, K. N.: On depolarization of visible light from water clouds for a monostatic lidar, J. Atmos. Sci., 29, 1000-1003, 1972.

Liou, K.-N. and Schotland, R. M.: Multiple Backscattering and Depolarization from Water Clouds for a Pulsed Lidar System, J. Atmos. Sci., 28, 772-774, 1971.

Liu, B. and Wang, Z.: Improved calibration method for depolarization lidar measurement, Opt. Express, 21, 14583-14590, 2013.

Mariani, Z., Strong, K., Wolff, M., Rowe, P., Walden, V., Fogal, P. F., Duck, T., Lesins, G., Turner, D. S., Cox, C., Eloranta, E., Drummond, J. R., Roy, C., Turner, D. D., Hudak, D., and Lindenmaier, I. A.: Infrared measurements in the Arctic using two Atmospheric Emitted Radiance Interferometers, Atmos. Meas. Tech., 5, 329-344, https://doi.org/10.5194/amt-5329-2012, 2012.

McCullough, E. M.: A new technique for interpreting depolarization measurements using the CRL atmospheric lidar in the Canadian High Arctic, $\mathrm{PhD}$ thesis, The University of Western Ontario, London, Ontario, Canada, 2015.

McCullough, E. M., Sica, R. J., Drummond, J. R., Nott, G., Perro, C., and Duck, T. J.: Three-channel single-wavelength lidar depolarization calibration, Atmos. Meas. Tech. Discuss., https://doi.org/10.5194/amt-2017-328, in review, 2017.

McGill, M., Hlavka, D., Hart, W., Scott, V. S., Spinhirne, J., and Schmid, B.: Cloud physics lidar: Instrument description and initial measurement results, Appl. Optics, 41, 3725-3734, 2002.

Mishchenko, M. I. and Hovenier, J. W.: Depolarization of light backscattered by randomly oriented nonspherical particles, Opt. Lett., 20, 1356-1358, 1995.

Moran, K. P., Martner, B. E., Post, M. J., Kropfli, R. A., Welsh, D. C., and Widener, K. B.: An Unattended Cloud-Profiling Radar for Use in Climate Research, B. Am. Meteorol. Soc., 79, 443455, 1998.

Mueller, H.: Development of a photoelastic shutter for modulating infrared light at audio frequencies, Tech. Rep. Report No. 16.4-1, 
OEMsr-579, MIT Research Project DIC-6085, Massachusetts Institute of Technology, Cambridge, Massachusetts, United States of America, 1943a.

Mueller, H.: The polarization optics of the photoelectric shutter, Tech. Rep. Report No. 16.4018. OEMsr-576; MIT Research Project DIC-6085, Massachusetts Institute of Technology, Cambridge, Massachusetts, United States of America, 1943b.

Mueller, H.: Lectures. Course 8.262, 1946a.

Mueller, H.: Theory of Polarimetric Investigations of Light Scattering. Parts I and II, Tech. Rep. Contract W-18-035-CWS-1304. D.I.C 2-6467, Massachusetts Institute of Technology, Cambridge, Massachusetts, United States of America, 1946b.

Mueller, H.: The Foundation of Optics, Abstract 17 in: Proceedings Of The Winter Meeting Of The Optical Society Of America 17, Hotel Pennsylvania, New York, New York, J. Opt. Soc. Am., 38, p. 661,1948 .

Neely III, R. R., Hayman, M., Stillwell, R., Thayer, J. P., Hardesty, R. M., O'Neill, M., Shupe, M. D., and Alvarez, C.: Polarization lidar at Summit, Greenland, for the detection of cloud phase and particle orientation, J. Atmos. Ocean. Tech., 30, 1635-1655, 2013.

Noel, V., Chepfer, H., Ledanois, G., Delaval, A., and Flamant, P. H.: Classification of particle effective shape ratios in cirrus clouds based on the lidar depolarization ratio, Appl. Optics, 41, 42454257, 2002.

Nott, G., Duck, T., Doyle, J., Coffin, M., Perro, C., Thackray, C., Drummond, J., Fogal, P., McCullough, E., and Sica, R.: A remotely operated lidar for aerosol, temperature, and water vapor profiling in the High Arctic, J. Atmos. Ocean. Tech., 29, 221234, 2012.

Pal, S. R. and Carswell, A. I.: Polarization properties of lidar backscattering from clouds, Appl. Optics, 12, 1530-1535, 1973.

Parke III, N. G.: Matrix Optics, PhD thesis, Massachusetts Institute of Technology, Cambridge, Massachusetts, United States of America, 1948.

Platt, C.: Lidar observation of a mixed-phase altostratus cloud, J. Appl. Meteorol., 16, 339-345, 1977.

Sassen, K.: The polarization lidar technique for cloud research: a review and current assessment, B. Am. Meteorol. Soc., 72, 18481866, 1991.

Sassen, K.: Lidar: range-resolved optical remote sensing of the atmosphere, chap. Polarization in lidar, no. 2 in Springer Series in Optical Sciences, Springer Science+Business Media Inc., Springer-Verlag, New York, 2005.
Sassen, K. and Benson, S.: A midlatitude cirrus cloud climatology from the Facility for Atmospheric Remote Sensing. Part II: Microphysical properties derived from lidar depolarization, J. Atmos. Sci., 58, 2103-2112, 2001.

Sassen, K. and Petrilla, R. L.: Lidar depolarization from multiple scattering in marine stratus clouds, Appl. Optics, 25, 1450-1459, 1986.

Schotland, R., Sassen, K., and Stone, R.: Observations by lidar of linear depolarization ratios for hydrometeors, J. Appl. Meteorol., 10, 1011-1017, 1971.

Serreze, M. C. and Barry, R. G.: Processes and impacts of Arctic amplification: A research synthesis, Global Planet. Change, 77, 85-96, 2011.

Shupe, M., Daniel, J., de Boer, G., Eloranta, E., Kollias, P., Long, C., Luke, E., Turner, D., and Verlinde, J.: A focus on mixed-phase clouds: The status of ground-based observational methods, B. Am. Meteorol. Soc., 89, 1549-1562, https://doi.org/10.1175/2008BAMS2378.1, 2008.

Shupe, M. D.: Clouds at Arctic Atmospheric Observatories. Part II: Thermodynamic Phase Characteristics, American Meteorological Society, 50, 645-661, https://doi.org/10.1175/2010JAMC2468.1, 2011.

Snels, M., Cairo, F., Colao, F., and Donfrancesco, G. D.: Calibration method for depolarization lidar measurements, Int. J. Remote Sens., 30, 5725-5736, 2009.

Spinhirne, J. D., Hansen, M. Z., and Caudill, L. O.: Cloud top remote sensing by airborne lidar, Appl. Optics, 21, 1564-1571, 1982.

Sun, Z. and Shine, K. P.: Studies of the radiative properties of ice and mixed phase clouds, Q. J. Roy. Meteor. Soc., 120, 111-137, 1994.

van de Hulst, H. C.: Light scattering by small particles, John Wiley and Sons, Inc., New York, 1957.

Venable, D. D., Whiteman, D. N., Calhoun, M., Dirisu, A. O., Connell, R., and Landulfo, E.: A lamp mapping technique for independent determination of the water vapor mixing ratio calibration factor for a Raman lidar system, Appl. Optics, 50, 4622-4632, 2011.

Wang, Z., Liu, D., Zhou, J., and Wang, Y.: Experimental determination of the calibration factor of polarization-Mie lidar, Opt. Rev., 16, 566-570, 2009.

Westbrook, C. D. and Illingworth, A. J.: The formation of ice in a long-lived supercooled layer cloud, Q. J. Roy. Meteor. Soc., 139, 2209-2221, 2013. 